Cómo citar este trabajo: Montoro-Gurich, C., \& Pons Izquierdo, J. J. (2021). The demographic aging of Spanish urban areas (2002-2017). Boletín de la Asociación de Geógrafos Españoles, (90). https://doi.org/10.21138/bage.3057

\title{
El envejecimiento demográfico de las áreas urbanas españolas (2002-2017)
}

The demographic aging of Spanish urban areas (2002-2017)

\section{Carolina Montoro-Gurich}

\author{
cmontoro@unav.es \\ Juan José Pons Izquierdo \\ jpons@unav.es \\ Departamento de Historia, Historia de Arte y Geografía \\ Universidad de Navarra (España)
}

\section{Resumen}

Este trabajo analiza y representa cartográficamente en cuatro momentos temporales (2002, 2007, 2012 y 2017) el envejecimiento de las áreas urbanas españolas y su relación con el envejecimiento de la provincia en la que están situadas. También se definen cuatro modelos de envejecimiento según la relación entre la intensidad del envejecimiento en 2020 y la tasa de variación del período completo. La Estadística del Padrón Continuo se emplea para el cálculo del porcentaje de población de 65 y más años -indicador de envejecimiento- y el de 80 y más años - de sobrenvejecimiento-. La delimitación del espacio urbano elegida es la del Atlas Estadístico de las Áreas Urbanas en España. Como principales conclusiones, (1) se han reducido los contrastes del envejecimiento entre el mundo urbano y el mundo rural, mientras que han aumentado los del sobrenvejecimiento a favor del mundo rural; (2) los contrastes más acusados los encontramos entre la mayoría de las áreas urbanas del noroeste español, con niveles altos y ritmo rápido de envejecimiento, frente a las áreas urbanas del litoral mediterráneo y andaluz, con niveles y ritmos más lentos de envejecimiento. 
Palabras clave: envejecimiento; sobrenvejecimiento; áreas urbanas.

\begin{abstract}
This work analyzes and cartographically represents ageing in Spanish urban areas and its relation to ageing processes in the province they are located in four time periods (2002, 2007, 2012 and 2017). Likewise, it defines four models of ageing according to the link between the intensity of ageing in the first analyzed year and the variation rate during the whole period. Population over-ageing goes through the same analysis. The Statistics of the Extended Census are utilized for the calculation of the percentage of population over 65 years - ageing indicator - and 80 years and beyond -over ageing-. The selected urban space delimitation belongs to the Statistic Atlas of Urban Areas of Spain. As main conclusions, (1) the contrasts of ageing between urban and rural areas have reduced, whereas they have increased in favor of the rural world. (2) The most acute contrasts are between most of the urban areas of northwestern Spain, displaying a high and fast-paced level of ageing, and the slow-paced, lower levels of ageing of the urban areas of the Mediterranean and Andalusian coasts.
\end{abstract}

Key words: ageing; over-ageing; urban areas.

\title{
1 Introducción
}

El proceso de modernización de las sociedades se traduce, demográficamente hablando, en el cambio desde niveles altos de mortalidad y natalidad a niveles bajos en ambos parámetros, lo que ha recibido el nombre de "Primera Transición Demográfica" (Casterline, 2003). En la actualidad, las poblaciones de los países occidentales se caracterizan por ser longevas -una gran mayoría de las personas llegan a edades avanzadas- y presentar unos niveles de fecundidad muy bajos, al nacer muy pocos niños por cada mujer en edad de tenerlos. Este alargamiento de la esperanza de vida al conjunto de la sociedad, así como el declive pronunciado y prolongado de la fecundidad, que ha limitado el tamaño de las nuevas generaciones (Pérez Díaz \& Abellán García, 2018), tiene como resultado el "envejecimiento demográfico". Se trata de sociedades en las que hay un volumen y un peso porcentual de personas mayores (65 y más años) y muy mayores (80 y más años) elevado, que además se sabe va a seguir aumentando. Esta transformación de la estructura por edades de las poblaciones es una de las consecuencias de la Primera Transición Demográfica, y al mismo tiempo, un factor definitorio de la llamada "Segunda Transición Demográfica" (Reques, 2009). En términos generales, la Segunda Transición Demográfica trae nuevos desafíos sociales, 
incluidos los relacionados con el incremento del envejecimiento, una menor estabilidad de los hogares y la integración de inmigrantes y otras culturas (Lesthaeghe, 2014).

El envejecimiento demográfico de España se inicia en la década de los setenta del siglo XX y se expande de forma constante, acelerándose desde mediados de los ochenta y en los noventa, para suavizarse con la llegada de migración extranjera, en la primera década del siglo XXI (Gurría Gascón, Reques Velasco \& Rodríguez Rodríguez, 2008; Reques Velasco, 2017). Según datos del Padrón Continuo (INE), a 1 de enero de 2017 había 8764204 personas mayores (65 y más años), un 18,8 \% sobre el total de la población. Esta cifra estaba todavía un poco por debajo de la media europea, que se situaba ese mismo año en el 19,5 \% (EUROSTAT, 2020). A su vez, los adultos mayores de 80 y más años representaban el 6,1% del volumen total de residentes en España. Este grupo seguirá ganando importancia entre la población mayor, lo que explica que en la actualidad se haga un gran hincapié en mejorar la calidad de vida en este período vital y se emplee el paradigma de "envejecimiento activo", tanto en la investigación como en las políticas públicas. El envejecimiento activo hace referencia al proceso de optimización de las oportunidades de salud, participación y seguridad con el fin de mejorar la calidad de vida a medida que las personas envejecen (World Health Organization, 2002, p. 12). La calidad de vida se traduce en que pueda mantener o mejorar sus condiciones vitales con el objeto de prolongar al máximo el tiempo de vida autónoma e independiente (Rojo Pérez \& Fernández Mayoralas, 2018).

El aumento de personas de 65 y más años representa, por un lado, el éxito social sobre la muerte y la enfermedad y la eficiencia de la reproducción humana (la 'revolución reproductiva' en palabras de Pérez Díaz, 2010). Por otro lado, abre un reto de justicia social y sostenibilidad para lograr atender a un número creciente de población vulnerable por razón de su edad (García Ballesteros \& Jiménez Blasco, 2016).

¿Qué importancia tiene el fenómeno migratorio en el proceso de envejecimiento demográfico? Resulta básico para entender los contrastes regionales en la distribución geográfica del envejecimiento. En España a partir de los años treinta del siglo XX se consolidan las corrientes migratorias con destino a los centros urbanos (García Docampo \& Otero, 2012), que se acentúan desde los años cincuenta y se prolongan hasta la crisis del petróleo de mediados de los setenta. El análisis del crecimiento de la población a nivel municipal revela que entre 1950 y 1981 se produjo una intensísima despoblación de la España rural, cuyo principal resultado es el envejecimiento y la desvitalidad demográfica actual y que, territorialmente, produjo una 
progresiva y marcada polarización rural-urbana. En ese período la España rural empezó a despoblarse, de forma tanto más intensa cuanto más marginal y de montaña eran sus municipios, debido a la masiva migración de personas jóvenes. La España urbana, en lógica correspondencia, creció, si bien de forma desigual en el territorio (Vinuesa Angulo, 1996; de Cos Guerra \& Reques Velasco, 2006). Al aumento generalizado de la población de las áreas urbanas de los años setenta le sucedió la ralentización y la aparición de distintas situaciones en los ochenta: entre el decrecimiento de las áreas afectadas por los procesos de reconversión industrial (El Ferrol, Bilbao, San Sebastián); y el crecimiento de las áreas beneficiadas por el turismo residencial, como Tenerife-Sur, Blanes-Lloret, Torrevieja o Costa Blanca (Gil-Alonso \& Bayona-i-Carrasco, 2012).

Por otro lado, la transformación de España en país de inmigración desde los años noventa del siglo XX y, especialmente, el fuerte incremento en los saldos migratorios positivos producido entre 2000 y 2008 tuvo un efecto rejuvenecedor, gracias a que se trataba de personas jóvenes y en edad de tener descendencia. Este influjo positivo de la inmigración no es exclusivo de un país, sino que se ha documentado en otros muchos contextos (Lewandowska-Gwarda \& Antczak, 2020), en particular en los países europeos más desarrollados (Antczak \& LewandowskaGwarda, 2019). Dentro de ese ámbito, en España fue especialmente relevante y contribuyó de forma decisiva a que a lo largo del período 2002-2017 el ritmo de envejecimiento poblacional haya sido inferior al de la media de los países europeos (EUROSTAT, 2020).

Este efecto, temporal, abundó en los contrastes regionales del envejecimiento demográfico, ya que no todas las regiones atrajeron con igual intensidad a la población inmigrante (Goerlich et al., 2007a, 2007b). Así, las regiones ubicadas en la cornisa cantábrica, Castilla y León y Extremadura fueron las menos beneficiadas por la recuperación demográfica asociada a la inmigración exterior, frente a otras áreas en las que el fenómeno tuvo mayor importancia, como Madrid y su entorno, la costa mediterránea, el eje del Ebro y los archipiélagos (Delgado Urrecho \& Martínez Fernández, 2016; Pujadas Rúbies \& Bayona-i-Carrasco, 2017; Otero-Enríquez et al., 2019).

Sin embargo, los contrastes más marcados se encuentran entre el mundo urbano y el rural. Este último, caracterizado por entidades de población pequeñas y envejecidas, se benefició durante los primeros años del siglo XX del impacto de la migración internacional (Morén-Alegret \& Solana, 2004; Collantes et al., 2010), aunque dicho impacto fue de relativa poca importancia (Collantes et al., 2014) y, además, se truncó con la llegada de la crisis (Roquer \& Blay, 2017). 
Frente a los espacios rurales, las ciudades españolas experimentaron un notable crecimiento demográfico gracias a la llegada de inmigrantes extranjeros. Esta migración, que buscó -en primer lugar - asentarse en los grandes centros urbanos (que reunían tanto las mayores oportunidades laborales, como las condiciones más favorables en cuanto a disponibilidad de vivienda y transporte público), terminó impactando en el proceso de suburbanización de las ciudades españolas (Bayona et al., 2018). El fenómeno que se describe no afectó solamente a las áreas metropolitanas más importantes, sino que incluyó también a las ciudades de tamaño medio y a las capitales de provincia, que desarrollaron espacios periurbanos cada vez más extensos (Reques Velasco, 2017).

La llegada de la crisis económica tuvo como consecuencia que los saldos migratorios volvieron a ser negativos y que, además, el crecimiento demográfico se estancó; desde el máximo de población registrado en el Censo de 2011, parecía que España iniciaba el declive de su población (Abellán García, Ayala García \& Pujol Rodríguez, 2017). Además, el cambio de ciclo migratorio se tradujo, también, en nuevas pautas de movilidad de la población inmigrada, que dejando los escenarios rurales se dirige a los entornos urbanos, revelando cuán frágil era la idea de recuperación demográfica del campo gracias a la inmigración (Otero-Enríquez et al., 2019).

Sin embargo, desde 2016 el país ha vuelto a experimentar un leve crecimiento poblacional gracias, fundamentalmente, a los saldos migratorios positivos. Así, en 2016 el saldo vegetativo fue, prácticamente, nulo; mientras que en 2017, 2018 y 2019 ha sido positivo y cada vez más importante, superando el último año las 500000 personas (INE, 2017, 2018, 2019, 2020).

La distribución geográfica del envejecimiento en España refleja el proceso histórico de concentración de la población: "de la montaña al valle, del interior hacia la costa, del campo a la ciudad y a las áreas metropolitanas" (Goerlich et al., 2015, p. 156). La tendencia al abandono de las áreas interiores del país con dirección hacia las franjas costeras y la capital todavía continúa en la actualidad, lo que se traduce en que todas las provincias que han ganado población en las últimas décadas son costeras o bien se sitúan alrededor de Madrid.

La persistencia en el tiempo de esta tendencia ha dejado como una de sus consecuencias un mundo rural cuyo el envejecimiento es claramente más importante que el de las ciudades (Delgado Urrecho \& Martínez Fernández, 2017). Esto es particularmente evidente en los municipios más pequeños del país. En el siguiente gráfico (Figura 1) se aprecia que existe una relación muy clara entre el número de habitantes y el porcentaje de personas mayores de 64 
años: cuanto más pequeño es el volumen de población del municipio, más posibilidades existen de encontrar un elevado envejecimiento demográfico.

Figura 1. Porcentaje de personas mayores (65 años) en municipios de hasta 2000 habitantes, según tamaño poblacional del municipio (2017)

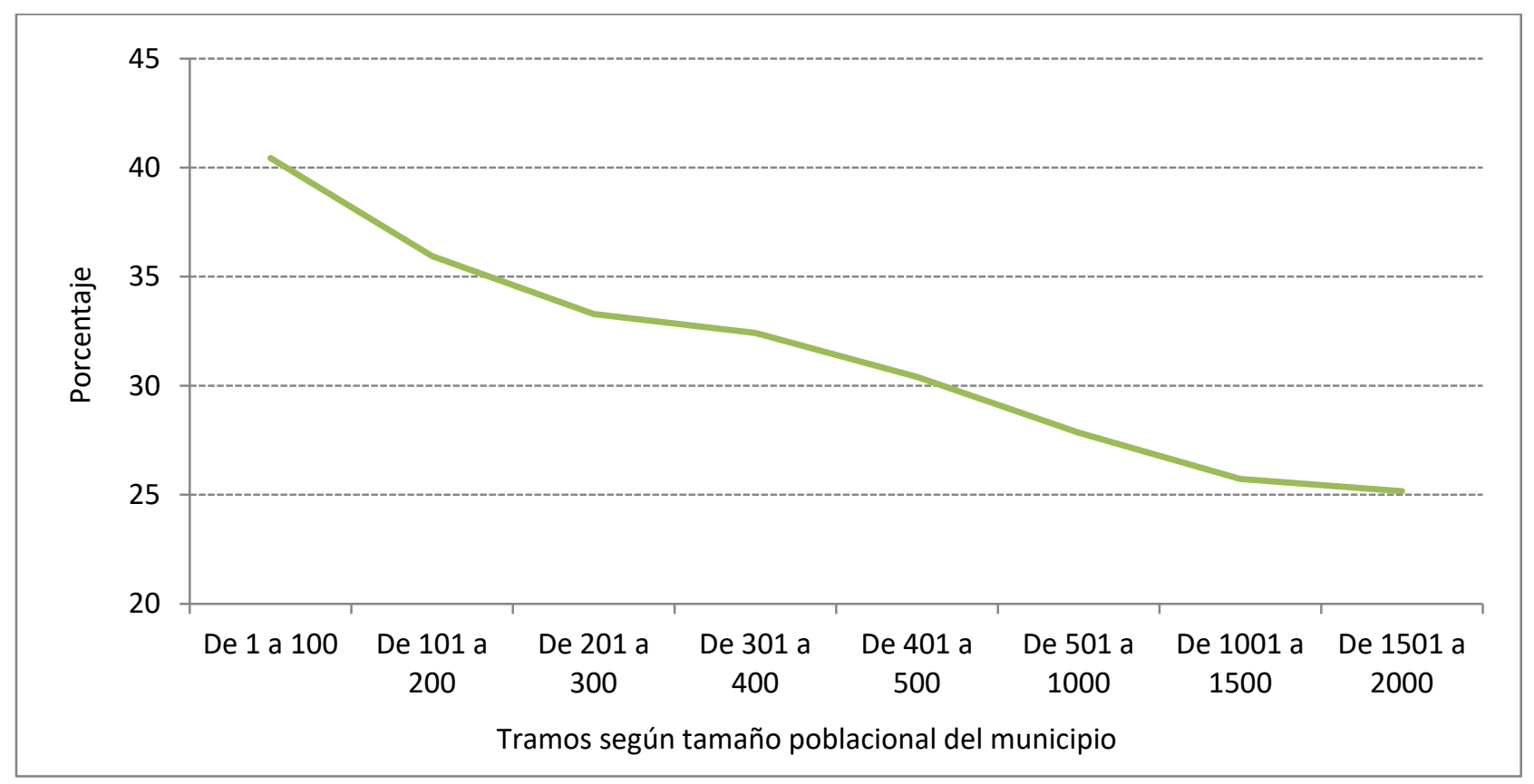

Fuente: elaboración propia a partir de los datos del Padrón Municipal de Habitantes de 2017

Según datos del Padrón Continuo (INE) a 1 de enero de 2017, el número de personas que vivían en municipios de menos de 2000 habitantes era de 2728017 (el 5,9 \% del total de población en España), de las cuales 28,3 \% tenían 65 y más años y, 11,6 \% más de 80.

Estas cifras ponen de manifiesto otro aspecto de la realidad que no conviene olvidar: aunque el envejecimiento sea porcentualmente más intenso en el mundo rural, el principal volumen de personas mayores se encuentra localizado en las áreas urbanas.

Dado que el envejecimiento varía a lo largo del tiempo (Garrocho \& Campos, 2015) y también, de acuerdo con el contexto demográfico en el que se inserta, cabe preguntarse: ¿cómo es el envejecimiento de las áreas urbanas españolas en los últimos años?, ¿cabe identificar niveles de envejecimiento provinciales a partir del envejecimiento de las áreas urbanas?, ¿hasta qué punto existen contrastes entre la vitalidad demográfica de un área urbana y la de la provincia en la que se sitúa?, ¿qué efectos ha tenido la inmigración de comienzos de siglo y en qué áreas urbanas se ha centrado? 
El objetivo de este trabajo es responder a estas y otras preguntas a través del análisis del porcentaje de personas de 65 y más años y de 80 y más años que viven en áreas urbanas, en cuatro momentos temporales (2002, 2007, 2012 y 2017). En esto coincide con la recomendación realizada por el Grupo Europeo de Cooperación Territorial ESPON/ACPA (2019) sobre la necesidad de profundizar en la investigación de la dimensión urbana del envejecimiento poblacional.

\section{Fuentes y metodología}

La realización del presente estudio se ha llevado a cabo con información de la población española por edad y sexo extraída de la Estadística del Padrón Continuo (INE, 2018), correspondiente a los años 2002, 2007, 2012 y 2017 y que abarca, por tanto, un período de 15 años. Con esta información se han elaborado los indicadores de la proporción de personas de 65 y más años y la proporción de personas de 80 y más años en cada una de las fechas señaladas. Se trata de los indicadores más utilizados en los estudios y las estadísticas oficiales sobre envejecimiento de la población (EUROSTAT, 2017; IMSERSO, 2016; ARUP, 2015).

La realidad es que la mejora en las condiciones de vida de la población más allá de un umbral "tradicional", asociado a la edad en la que las personas llegan a la jubilación, bien merece una reflexión sobre el significado de ser una persona mayor en la actualidad. En el ámbito demográfico, por ejemplo, se ha planteado para señalar el inicio de la vejez un nuevo indicador, la edad prospectiva. Teniendo como referencia la muerte se define como el momento en el que la gente tiene una edad en la que su esperanza de vida, o vida restante, es de 15 años. Se plantea que, de esta forma, se recogería mejor los progresos en estas condiciones y de su esperanza de vida, creciente desde hace muchas décadas y con perspectiva de seguir aumentando (Ramiro Fariñas, Pujol Rodríguez \& Abellán García, 2016).

En efecto, el límite de 65 años - que define una persona como mayor en estos estudios y estadísticas- no refleja las mejoras en las condiciones de salud o el incremento de la esperanza de vida acaecidos en las últimas décadas; pero se sigue utilizando porque es fácil de calcular y su gran difusión facilita análisis comparativos. Por otro lado, el límite de 80 años para medir el sobrenvejecimiento está relacionado con el hecho de que a partir de esa edad aumenta claramente el volumen de personas con limitaciones funcionales (Abellán García, Ayala García \& Pujol Rodríguez, 2017). 
El cálculo de estos indicadores, en los que se pretende diferenciar el carácter urbano o rural de la población mayor, requiere de una delimitación de las ciudades o áreas metropolitanas españolas. Esto no es una tarea ni mucho menos sencilla, dados los diferentes criterios metodológicos que pueden emplearse para ello (Feria Toribio, 2004; Boix Domènech, 2007; Roca Cladera, Moix Bergadà \& Arellano Ramos, 2012).

Por este motivo y con el fin de no distraer innecesariamente del objetivo primordial de este trabajo, se asume la delimitación del espacio urbano aportada en el Atlas Estadístico de las Áreas Urbanas en España (Ministerio de Fomento, 2017), conformada por 754 municipios, agrupados en 86 grandes áreas urbanas (Figura 2).

Como puede verse, se trata de un número relativamente pequeño de municipios (el 9,2 \% del total) que, sin embargo, alberga a más de dos tercios de la población del país: 32 millones de personas. El resto de circunscripciones municipales, incluyendo las que esa misma fuente considera "pequeñas áreas urbanas" y que se encuentran situadas en un umbral de población entre los 5000 y 50000 habitantes, se han considerado rurales a efectos de este trabajo. En total son 7451 municipios y en ellos residen más de 14 millones de habitantes.

Además de los porcentajes de envejecimiento y sobreenvejecimiento, en este trabajo se calcula la variación porcentual de ambos indicadores entre los años analizados y para el conjunto del período. Por otro lado, poniendo en relación el envejecimiento (y sobreenvejecimiento) demográfico al inicio, en 2002, y la tasa de variación mostrada en el total del período -2002 a 2017-, se establece una modelización del envejecimiento y sobreenvejecimiento de las áreas urbanas españolas clasificándolas en cuatro categorías. Finalmente, se analiza la relación entre envejecimiento y población inmigrante en cada una de estas categorías de área urbana con los coeficientes de correlación. Para evitar, en la medida de lo posible, explicaciones farragosas previas a la visualización de los datos a través de la cartografía y otras figuras, los detalles sobre la metodología empleada en cada caso se presentarán en los correspondientes epígrafes.

Finalmente, solo resta hacer una breve referencia a los programas informáticos utilizados: Microsoft Excel 2013 para el manejo de datos y la creación de gráficos y el sistema de información geográfica ArcGIS v. 10.5 de ESRI para realizar las representaciones cartográficas. 
Figura 2. Identificación de las áreas urbanas incluidas en el estudio, con su población en 2017

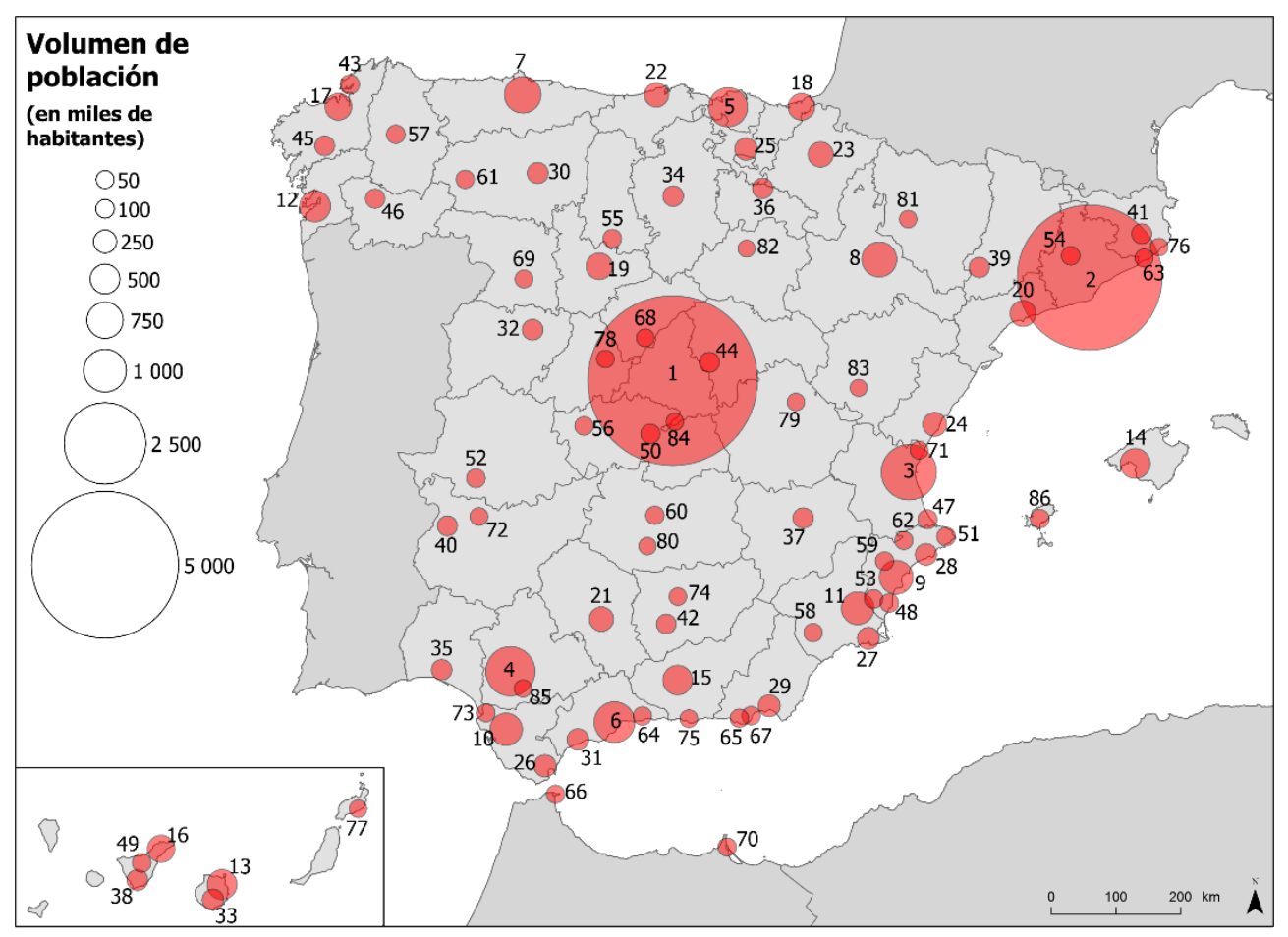

Leyenda: 1: Madrid; 2: Barcelona; 3: Valencia; 4: Sevilla; 5: Bilbao; 6: Málaga; 7: Asturias; 8: Zaragoza; 9: Alicante-Elche; 10: Bahía de Cádiz; 11: Murcia; 12: Vigo-Pontevedra; 13: Las Palmas de Gran Canaria; 14: Palma; 15: Granada; 16: Santa Cruz de Tenerife-La Laguna; 17: A Coruña; 18: Donostia/San Sebastián; 19: Valladolid; 20 : Tarragona-Reus; 21: Córdoba; 22: Santander-Torrelavega; 23: Pamplona; 24: Castellón de la Plana; 25: Vitoria/Gasteiz; 26: Bahía de Algeciras; 27: Cartagena; 28: Costa Blanca; 29: Almería; 30: León; 31: Costa del Sol; 32: Salamanca; 33: Gran Canaria Sur; 34: Burgos; 35: Huelva; 36: Logroño; 37: Albacete; 38: Tenerife Sur; 39: Lleida; 40: Badajoz; 41: Girona; 42: Jaén; 43: Ferrol; 44: Guadalajara; 45: Santiago de Compostela; 46: Ourense; 47: Gandía; 48: Torrevieja; 49: Valle de la Orotava; 50: Toledo; 51: Denia-ávea; 52: Cáceres; 53: Orihuela; 54: Manresa; 55: Palencia; 56: Talavera de la Reina; 57: Lugo; 58: Lorca; 59: Elda-Petrer; 60: Ciudad Real; 61: Ponferrada; 62: Alcoy/Alcoi; 63: Blanes-Lloret de Mar; 64: Vélez-Málaga; 65: El Ejido; 66: Ceuta; 67: Roquetas de Mar; 68: Segovia; 69: Zamora; 70: Melilla; 71: Sagunto/Sagunt; 72: Mérida; 73: Sanlúcar de Barrameda; 74: Linares; 75: Motril; 76: Sant Feliú de Guixols; 77: Arrecife; 78: Ávila; 79: Cuenca; 80: Puertollano; 81: Huesca; 82: Soria; 83: Teruel; 84: Aranjuez; 85: Utrera; 86: Eivissa.

Fuente: elaboración propia a partir del Atlas Estadístico de las Áreas Urbanas de España (2018) y del Padrón Municipal de Habitantes (2017)

\section{Los contrastes en el envejecimiento entre el mundo urbano y el rural}

En 2017 había 5,7 millones de personas de 65 y más años residiendo en ciudades, es decir un $18 \%$ del total de sus habitantes. A pesar de lo abultado de esta cifra, la población urbana es - 
en términos generales- bastante más joven que la rural. Tanto si se considera la proporción de personas de $\geq 65$ años como la de personas de 80 y más años, se aprecia que, en conjunto, el medio rural se sitúa siempre por encima de la media de envejecimiento del país y de la población agrupada en las áreas urbanas (Figura 3).

Figura 3. Evolución del porcentaje de población mayor (65 años) y muy mayor (80 años) en España (total y según ámbitos urbano y rural)
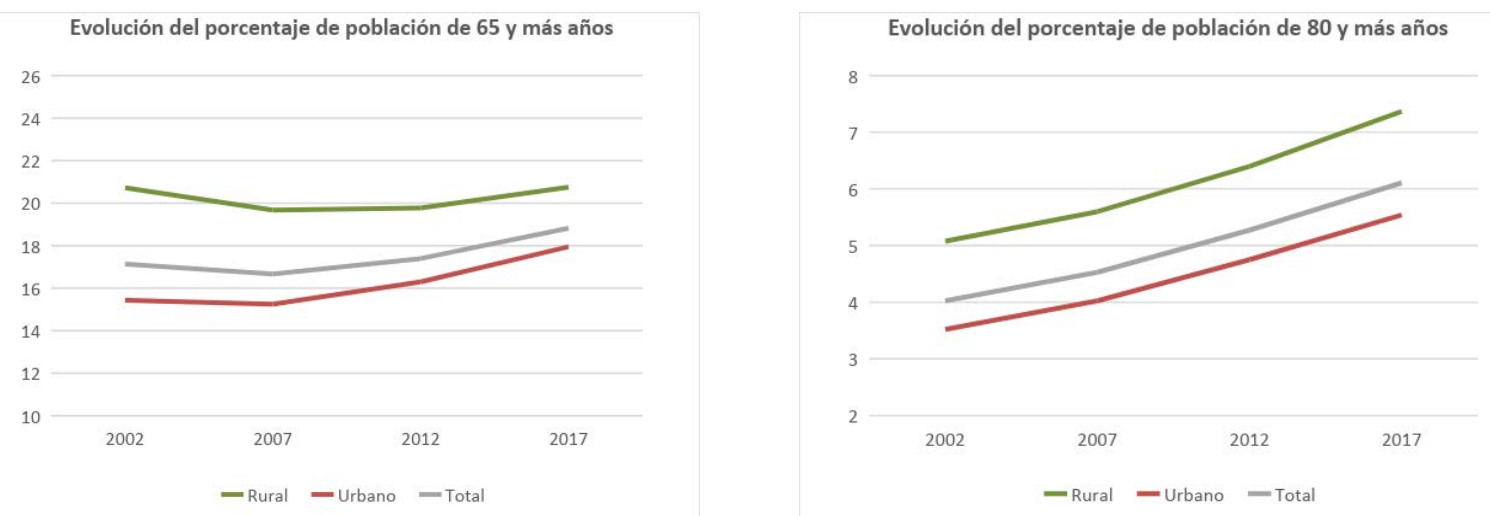

Fuente: elaboración propia a partir de los datos del Padrón Municipal de Habitantes

En el caso de las personas de 65 y más años (8,7 millones de habitantes en total), la brecha entre el mundo rural y el urbano ha disminuido de manera muy clara a lo largo del período estudiado: en 2002 era de casi 4,5 puntos y en la actualidad no llega a 3 puntos porcentuales. Esto significa que el proceso de envejecimiento ha sido más rápido en las ciudades, lo cual coincide con lo observado para el conjunto de Europa (Lewandowska-Gwarda \& Antczak, 2020).

Por el contrario, entre los mayores de 80 años (2,8 millones), aun habiendo menos diferencias de partida, la importancia relativa dentro del conjunto de la población ha crecido más rápido en el ámbito rural y, de esta manera, el diferencial con las áreas urbanas es un poco mayor en la actualidad (1,8 puntos).

Los dos gráficos anteriores permiten también observar que la evolución del envejecimiento de la población de las ciudades españolas no ha sido lineal. En el primer quinquenio de los estudiados (2002-2007) el porcentaje de personas de 65 y más años se redujo ligeramente, pasando del 15,4 \% al 15,2 \% de la población urbana. Este rejuvenecimiento de las ciudades se produjo, como ya ha sido señalado, en un contexto de fuerte crecimiento de la población debido al factor migratorio y a pesar de que el número de mayores residentes en ellas aumentó en más de 300000 personas. 
Otro indicador de envejecimiento es la edad media de la población. En 2017 la población española tenía una edad media de 42,9 años, casi 10 años más que en 1970, lo que supone un incremento muy significativo en este último medio siglo (Pérez Díaz \& Abellán García, 2018). Los contrastes entre el mundo rural y urbano español son también bastante significativos para este indicador: la edad media en el entorno rural es de 44,1 años en 2017, frente a los 42,4 años del ámbito urbano.

La Figura 4 muestra, empleando una pirámide de población, el perfil por sexo y entorno de residencia -urbano versus rural- de las personas de 60 y más años residentes en España y permite matizar esa mayor juventud de la población urbana en el año 2017. Las mujeres tienen un peso porcentual mayor que los hombres en las poblaciones de 60 y más años, residan éstas en un entorno rural o urbano. A este hecho - habitual- se puede añadir gracias a esta pirámide que los hombres tienen, para todas las cohortes, un mayor peso porcentual en el entorno rural que en el urbano salvo a partir de los 95 años. Entre las mujeres, sin embargo, las cohortes de entre 60 y 69 años son ligeramente menos importantes en el mundo rural que en el urbano, mientras que a partir de los 70 años el peso porcentual de las adultas mayores residentes en lo rural aumenta, para volver a ser muy semejante en ambos entornos a partir de los 95 años.

Figura 4. Perfil de las personas de 60 y más años por edad, sexo y entorno de residencia en España (2017)

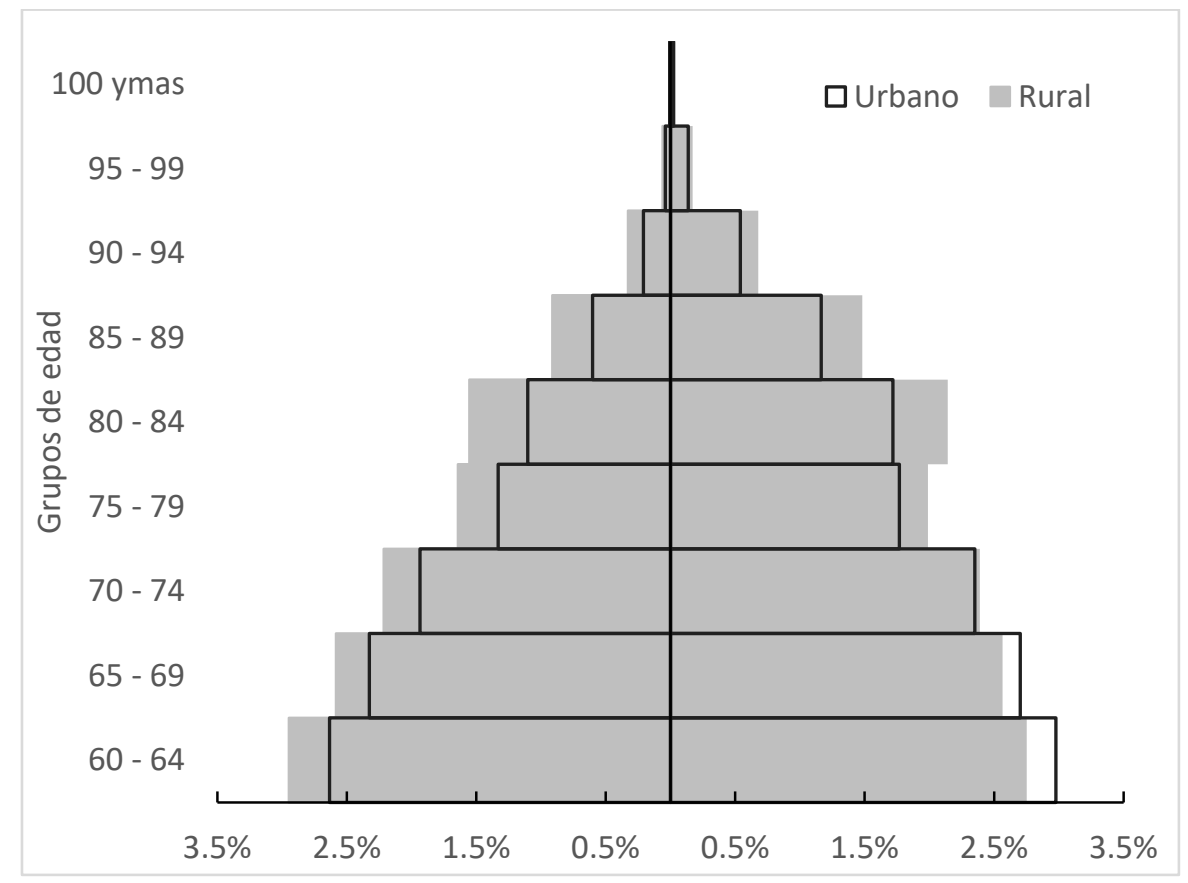

Fuente: elaboración propia a partir de los datos del Padrón Municipal de Habitantes. 
Si examinamos la relación de otra forma, empleando la tasa de feminidad, en 2017 encontramos en el mundo rural que entre la población de 65 y más años hay 1,2 mujeres por cada hombre, cifra que aumenta a 1,38 mujeres por cada hombre en el mundo urbano. Entre la población de 80 y más años se agudizan los contrastes: 1,57 mujeres por cada hombre en lo rural frente a 1,83 mujeres por cada hombre en lo urbano.

La diferencia que, a nivel general, se aprecia entre el envejecimiento del campo y de las ciudades, también es reconocible a una escala local. La comparación entre los porcentajes de personas mayores de las diferentes áreas urbanas y la de sus respectivas provincias resulta sumamente expresiva. Como era de esperar, los índices de envejecimiento de las ciudades han reflejado tradicionalmente valores inferiores a los de sus respectivas provincias y a los del total nacional (Vinuesa, 1996), debido al crecimiento migratorio al efecto rejuvenecedor que este suele traer a corto plazo.

Este modelo general sigue siendo válido actualmente, aunque bien es cierto que no se verifica en todos los casos. En el año 2017, el 82 \% de las áreas urbanas españolas (69) tenía un envejecimiento (porcentaje de personas de 65 y más años) inferior al de sus provincias; sin embargo, las 15 restantes (de un total de 84, pues aquí no se contabilizan las dos ciudades autónomas) presentaban una situación inversa (Figura 5).

Entre las primeras, destacan algunas de las áreas urbanas gallegas (Ourense y Lugo) y castellanoleonesas (Zamora, Ávila, Soria y León) que, a pesar de estar entre las más envejecidas de todo el país, tienen un diferencial superior o muy cercano a los 10 puntos porcentuales con respecto a la que presentan el resto de los municipios de sus provincias. Esta aparente paradoja se produce porque aun siendo capitales con un porcentaje de adultos mayores elevadísimo (salvo en un caso siempre por encima del $20 \%$ ), sus provincias lo están todavía mucho más (habitualmente, en cifras superiores al $30 \%$ ).

En esa parte alta de la tabla, que ocupan los casos con mayores contrastes, también se encuentran otras áreas urbanas cuya situación es bien distinta, dado que en este caso sí se hallan entre las ciudades más jóvenes de todo el país. Se trata de las áreas urbanas de Roquetas de $\operatorname{Mar}(8,5 \%)$, Tenerife Sur $(10,5 \%)$ y El Ejido $(8,7 \%)$, cuya distancia a sus provincias es también muy elevada. 
Figura 5. Comparación del envejecimiento de las áreas urbanas con el resto de las provincias en las que se ubican (2017)

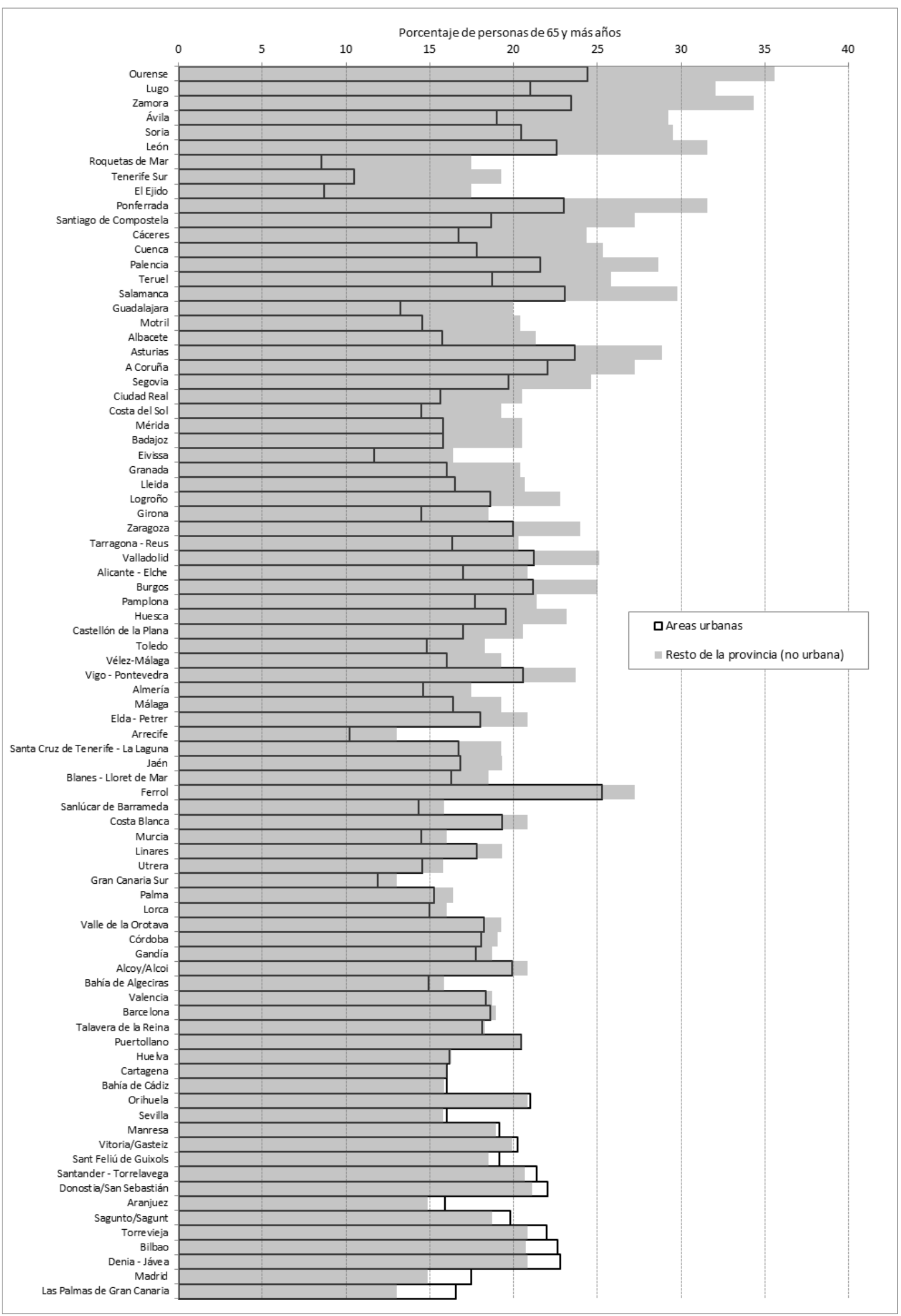

Fuente: elaboración propia a partir de los datos del Padrón Municipal de Habitantes (2017) 
En el extremo contrario de la tabla se sitúan las áreas urbanas envejecidas en comparación con el contexto territorial en el que se encuentran. De entre todas ellas destaca, por su importancia poblacional, la presencia de Madrid (17,5 \% de personas de 65 y más años en el área urbana y $14,9 \%$ en el resto de la provincia).

Sin embargo, no es la que mayor diferencial presenta, dado que ese puesto corresponde al área urbana de Las Palmas de Gran Canaria, cuyo diferencial con los municipios restantes de la provincia llega a los 3,5 puntos.

También aparecen como espacios urbanos más envejecidos que su entorno las tres capitales vascas y Santander, así como varias de las áreas del levante peninsular: Denia-Jávea y Torrevieja. Como se verá más adelante, los modelos de envejecimiento de estas últimas ciudades son bastante diferentes a los de la costa cantábrica.

Por último y ya casi en equilibrio entre la ciudad y la provincia, pero todavía con tasas de envejecimiento urbano superior, destacan dos casos andaluces: Bahía de Cádiz y Sevilla.

\section{Evolución del envejecimiento en las áreas urbanas}

En este apartado abordaremos separadamente la evolución del envejecimiento (personas de 65 y más años) y del sobrenvejecimiento (personas de 80 y más años), que si bien son bastante paralelas, en ocasiones permiten dibujar matices interesantes dentro del análisis de este fenómeno en el mundo urbano.

\section{1 Población de 65 y más años}

Iniciamos nuestro análisis contemplando los cambios en la proporción de personas de 65 y más años por áreas urbanas (Figura 6). En 2002 se podía dibujar una línea imaginaria con trazado noroeste (áreas urbanas de A Coruña, Santiago) - sureste (Alicante, Orihuela), que dejaba al norte de la misma a la gran mayoría de las áreas urbanas envejecidas. En concreto, Denia-jávea, Torrevieja, Ferrol, Asturias y Manresa presentan proporciones superiores al $20 \%$ de adultos mayores, frente a las de Roquetas de Mar y El Ejido -en la Península- y Arrecife, Tenerife Sur y Gran Canaria Sur -en las Islas Canarias-, con proporciones inferiores al $10 \%$ de personas de 65 y más años. 
Figura 6. Proporción de personas de 65 y más años en las áreas urbanas españolas
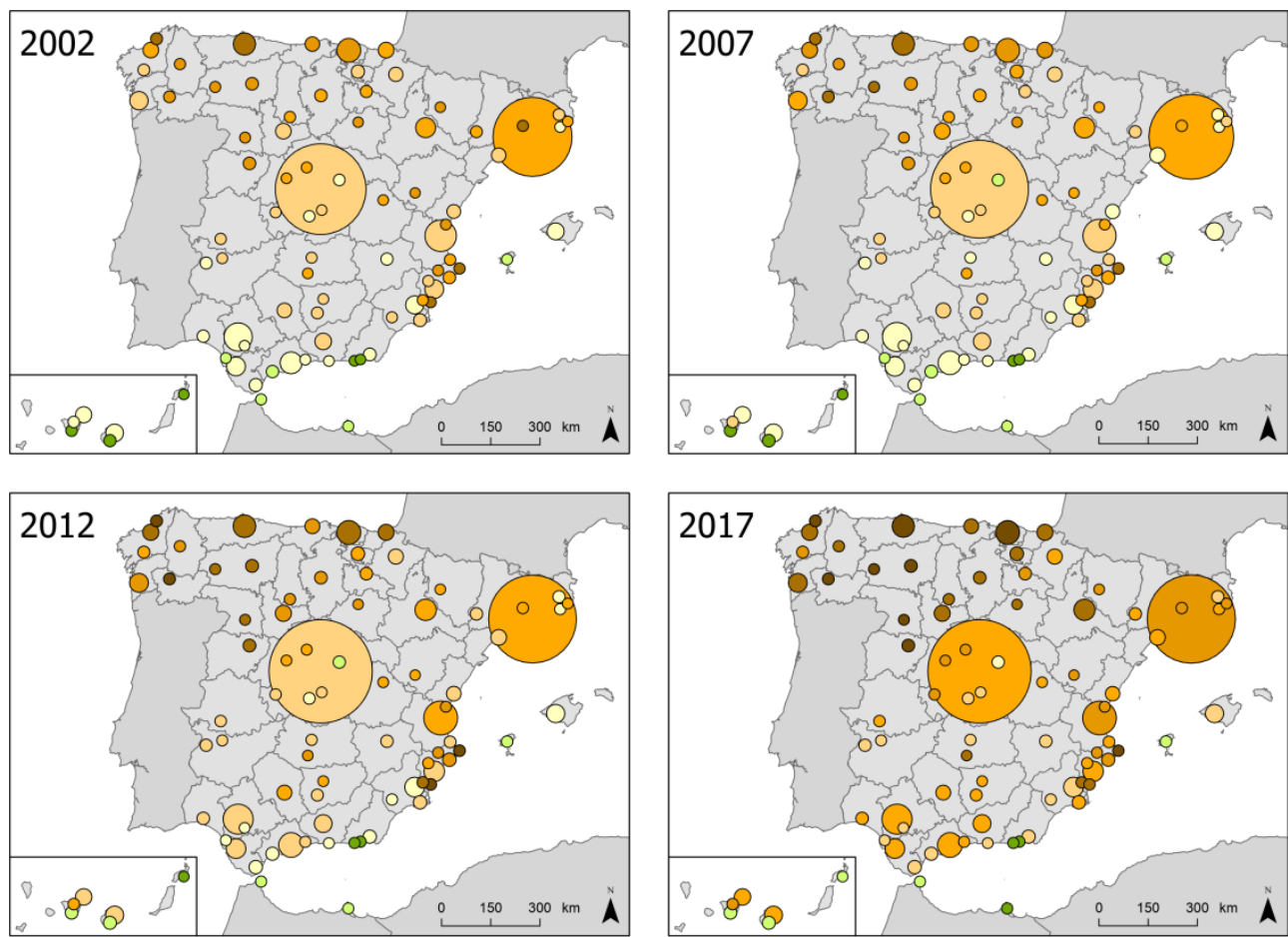

Volumen de población

(en miles de habitantes)

$505^{\circ} 5^{\circ} 5^{\circ} 1^{\circ} 2^{\circ} 2^{5^{\circ}} 5^{\circ}$
Población de 65 y más años

(en porcentaje sobre el total de la población)

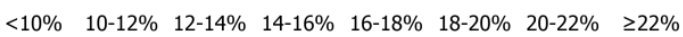

Fuente: elaboración propia a partir de los datos del Padrón Municipal de Habitantes

Cinco años más tarde, en 2007, la distribución geográfica de este indicador es bastante semejante, pero, es en la variación del porcentaje entre 2002 y 2007 (Figura 7) donde aparecen unos contrastes muy claros entre unas áreas urbanas y otras. Son numerosas las áreas urbanas que asisten a un rejuvenecimiento de su población, reflejado en la disminución de la proporción de personas de 65 y más años. Los considerables flujos positivos de inmigrantes están detrás de la importante caída del envejecimiento en Lleida, Girona, Manresa, Guadalajara, Aranjuez, Gandía y El Ejido (entre un 10 \% y un 20 \%) y algo menor (por debajo del 10 \%) en otras 44 áreas urbanas. Por el contrario, un total de 35 áreas urbanas ven aumentar el porcentaje de adultos mayores, si bien es casi siempre por debajo del $6 \%$, a excepción de Vitoria/Gasteiz, Orihuela, Las Palmas de Gran Canaria, Gran Canaria Sur y Valle de la Orotava. Tan solo en una de esas áreas urbanas, Tenerife Sur, el envejecimiento crece algo más del $10 \%$.

El año 2012 marca un cambio en el proceso de envejecimiento, pero no tanto en la localización geográfica de éste - las áreas urbanas más envejecidas son las mismas-, sino más bien en la intensidad del fenómeno (Figura 6). Por primera vez encontramos áreas urbanas con 
proporciones de personas de 65 y más años superiores al 22 \% del total de población: se trata de Ourense, Ferrol, Denia-ávea y Torrevieja. Y, en otras 9 más, porcentajes entre el 20 \% y 22 \% (A Coruña, Ponferrada, Zamora, León, Salamanca, Asturias, Bilbao, Donostia/San Sebastián y Orihuela). La línea que marca el eje del envejecimiento descrita en 2002 se mantiene, con la excepción del área urbana de Guadalajara, que desde 2007 se sitúa entre las más jóvenes.

El mapa de variación entre 2007 y 2012 (Figura 7) refleja el fin de la ilusión de rejuvenecimiento provocada por la inmigración y el cambio de tendencia de este fenómeno. Es un mapa en el que domina el tono rojizo, ya que prácticamente todas las áreas urbanas experimentan variaciones que se traducen en el incremento de la proporción de personas de 65 y más años, eso sí inferiores al 10 \%. Sin embargo, hay áreas urbanas en las que esta proporción se incrementa de forma más significativa, como los casos de Vigo-Pontevedra y Valladolid en el cuadrante noroeste y una serie de áreas urbanas en el litoral -tanto atlántico y mediterráneo- y en las Islas Canarias, con variaciones entre el $15 \%$ y el $20 \%$. Se trata de Gran Canaria Sur, Arrecife, Valle de la Orotava, Costa del Sol, Torrevieja, Orihuela y Denia-Jávea; y el caso extremo de Tenerife Sur, con un incremento del 20,7\%. En este período, solo 6 áreas urbanas ven disminuir, débilmente, la proporción de personas de 65 y más años; se trata de las catalanas Lleida, Manresa y Girona, a las que se suma Cuenca en el interior, así como Ceuta y Melilla.

El mapa correspondiente a 2017 aparece, prácticamente todo él en tonalidades cálidas (figura 6). Ya no cabe hablar de una línea o eje que vertebra los territorios con áreas urbanas más envejecidas con respecto a las más jóvenes, aunque el cuadrante noroeste presenta los porcentajes más elevados de población urbana de 65 y más años. Así, de las 12 áreas urbanas con proporciones de adultos mayores iguales o superiores al $22 \%, 10$ formaban parte de este cuadrante ya que eran gallegas (Ferrol, Ourense, A Coruña), castellano-leonesas (Zamora, Salamanca, León, Ponferrada) o del litoral cantábrico (Asturias, Bilbao, Donostia/San Sebastián). A ellas se suman Denia-Jávea y Torrevieja, áreas urbanas caracterizadas desde el comienzo del período analizado por una proporción importante de personas de 65 y más años, lógico habida cuenta su atractivo como áreas de "turismo residencial" desde los años ochenta-noventa del siglo $X X$, con inmigrantes de origen nacional e internacional. A sus características climáticas y de coste de vida se añade que en estos municipios se ha llevado a cabo una política activa de promoción de los usos residenciales, lo que ha facilitado que sean elegidos como lugar de retiro de jubilados europeos (Cutillas, 2009). Otro grupo de 11 áreas urbanas presenta valores de entre 
20 y 22 \% de población de 65 y más años, entre las que se encuentran Santander-Torrelavega, Palencia, Valladolid, Burgos, Lugo, etc.

En este panorama sobresalen, por representar los valores más bajos de envejecimiento (siempre inferiores al 10 \% del total de la población), los casos de Roquetas de Mar, El Ejido y Melilla, áreas en las que el perfil etario de los migrantes, de tipo económico, se caracteriza por su juventud.

Figura 7. Variación del porcentaje de personas de 65 y más años en las áreas urbanas españolas
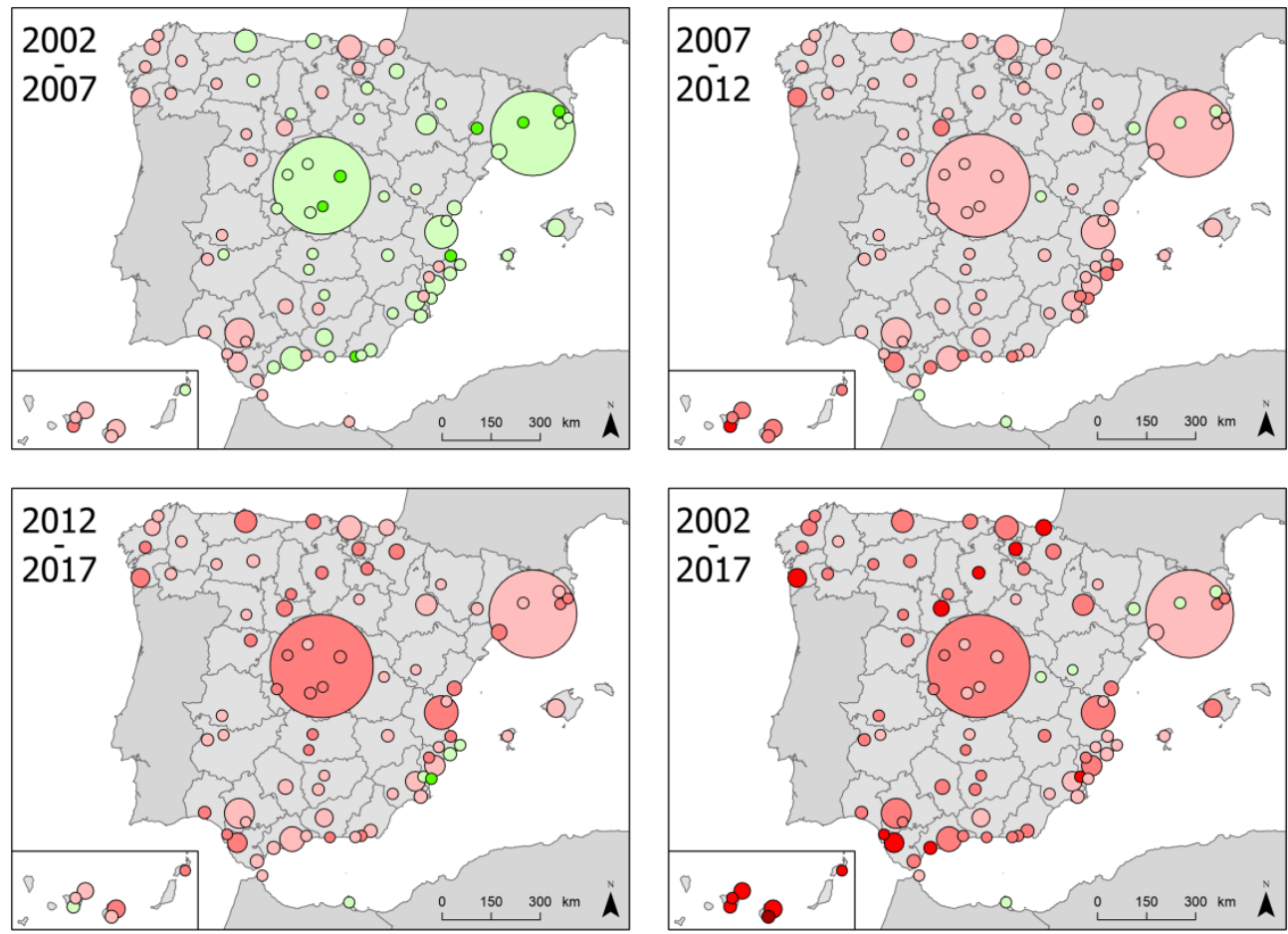

Volumen de población

(en miles de habitantes)

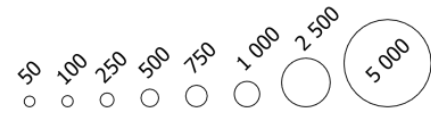

Variación de la población de 65 y más años

(porcentaje de cambio en el período indicado)

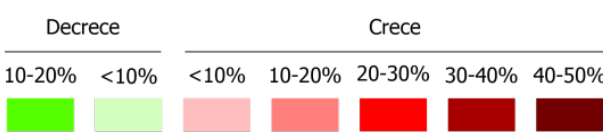

Fuente: elaboración propia a partir de los datos del Padrón Municipal de Habitantes

El mapa de variaciones entre 2012 y 2017 (Figura 7) es una prolongación del que veíamos para el período 2007-2012, si bien es claramente mayor el número de áreas urbanas que han aumentado el envejecimiento entre un $10 \%$ y un $20 \%$. Y un elemento sorpresivo: un pequeño grupo de áreas urbanas ubicadas - casi todas ellas- en la provincia de Alicante, que en este período reflejan un rejuvenecimiento. Se trata de Torrevieja, especialmente, y en menor medida, de Denia-ávea, Costa Blanca y Orihuela, además de Tenerife Sur y Melilla. 
Finalmente, el mapa que refleja la variación entre el comienzo y el final del período analizado -2002 en comparación con 2017 (Figura 7)- repite, en cuanto a distribución geográfica, el mapa de variaciones 2007-2012, pero con intensidades más acusadas. Un total de 15 áreas urbanas han visto crecer la proporción de población de 65 y más años en más de un 20 \%: Gran Canaria Sur (en un 33 \%), Tenerife Sur, Arrecife, Las Palmas de Gran Canaria y Valladolid (entre un $25 \%$ y un $30 \%$ ), seguidas por las áreas urbanas cuya proporción ha crecido entre un $20 \%$ y un 24,9\%, como Vigo-Pontevedra, Burgos, Vitoria/Gasteiz y Donostia/San Sebastián en la mitad septentrional de la Península; Sanlúcar de Barrameda, Bahía de Cádiz, Orihuela y Costa del Sol en la mitad meridional; y Valle de la Orotava y Santa Cruz de Tenerife-La Laguna en las Canarias. Otras 42 áreas urbanas presentan crecimientos de entre un $10 \%$ y un $20 \%$ y tan solo 6 áreas urbanas han visto disminuir estos porcentajes, si bien débilmente (por debajo del 10 \%): Melilla, Manresa, Lleida, Girona, Teruel y Cuenca.

A la vista de estos datos es evidente la gran extensión del envejecimiento urbano, algo que comparte con los demás países europeos, en los que, según algunos estudios (LewandowskaGwarda \& Antczak, 2020), en torno a un $93 \%$ de las ciudades se han visto en las últimas décadas afectadas por este fenómeno. Además, lejos de ser un hecho del pasado, las tendencias demográficas regresivas observadas en el conjunto del continente hacen prever que el envejecimiento de las poblaciones urbanas continuará en la misma línea durante los próximos años (Lauf, Haase \& Kleinschmit, 2016) o incluso se acentuará (Nader et al., 2018).

\subsection{Población de 80 y más años}

La proporción de personas de mayor edad en las áreas urbanas españolas ha aumentado también de forma sensible a lo largo del período analizado (Figura 8). Los porcentajes más elevados se situaban en 2002 entre un $5 \%$ y un 6 \% de la población, localizados sólo en 5 áreas urbanas: Ourense, Soria, Huesca, Teruel y Manresa. En 2007 ya había 29 áreas urbanas por encima del $5 \%$ y un tercio de estas se encontraban entre un $6 \%$ y un $8 \%$ de personas de edad muy avanzada. El proceso se intensifica con el paso del tiempo: en 2012 de 10 áreas urbanas se pasa a 18 y en 2017, se alcanzan las 23. Además de estas, otras 8 pasan al siguiente intervalo, el de más de un 8 \% de la población de 80 y más años. En este "grupo de cabeza" encontramos las áreas de Ourense, Ponferrada, Ferrol, Asturias, León, Zamora, Salamanca y Soria. 
La distribución geográfica del sobrenvejecimiento refleja la inercia demográfica de unas poblaciones beneficiadas por el alargamiento de la esperanza de vida, pero faltas de vitalidad por la escasa incorporación de nuevos efectivos, debido a nacimientos o inmigración.

Por otro lado, las áreas urbanas con proporciones más bajas de personas de 80 y más años se localizan en las islas Canarias y en la costa andaluza. En 2002, 2007 y 2012, 5 áreas urbanas tenían menos de un 2 \%: Tenerife, Gran Canaria, Arrecife, El Ejido y Roquetas de Mar; seguidas muy de cerca por Ceuta y Melilla. En 2017 sólo Roquetas de Mar permanece en la categoría de menos de un $2 \%$ de población de 80 y más años, pero todas las demás áreas citadas en este párrafo se sitúan en la categoría inmediatamente superior, entre $2 \%$ y 4 \%, junto a Sanlúcar de Barrameda, Bahía de Algeciras, Motril, Costa del Sol y Eivissa, que en años anteriores ya estaban en estos niveles. En estos casos, y a la espera de un estudio más detallado, cabría hablar de un efecto combinado de dos movilidades de carácter muy diferente: el atractivo para inmigrantes jóvenes, de tipo económico y la movilidad residencial de personas de edad avanzada, que al perder su independencia domiciliaria como consecuencia de la edad y necesitar una mayor atención, se podrían estar desplazando a otros municipios fuera de las áreas urbanas, ya fueran éstos el lugar de residencia de los hijos o el de ubicación de residencias para personas mayores (Pujadas Rúbies, Bayona-i-Carrasco \& Rubiales Pérez, 2016).

A la vista de los mapas que muestran el sobrenvejecimiento (Figura 8), ese eje noroeste-sureste que dejaba las áreas urbanas con mayores proporciones de personas de 64 y más años al norte que al sur del mismo se reproduce también para las personas de 80 y más años. 
Figura 8. Proporción de personas de 80 y más años en las áreas urbanas españolas
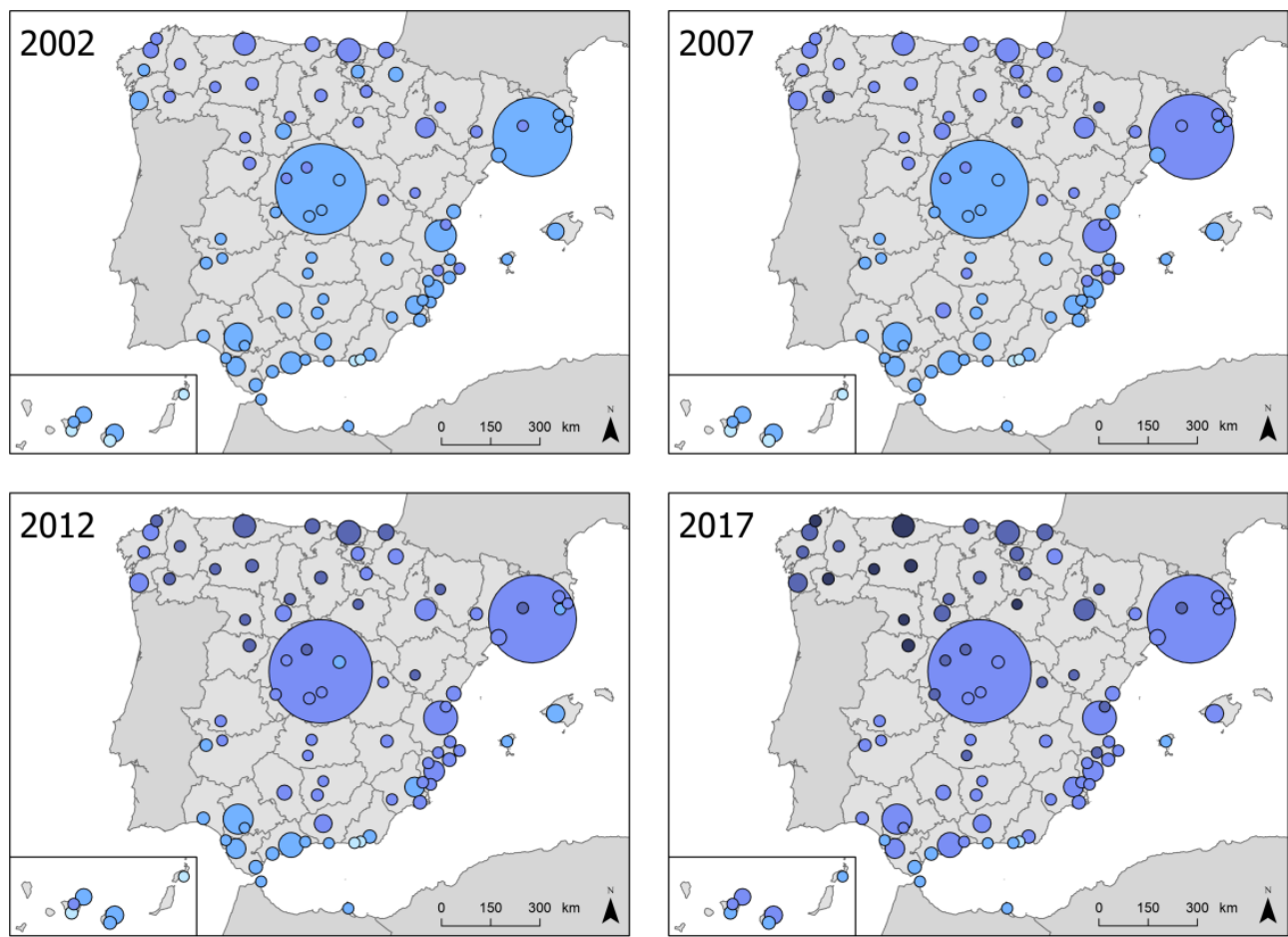

Volumen de población

(en miles de habitantes)

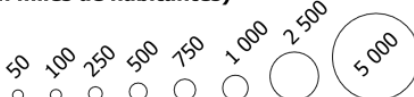

Población de 80 y más años (en porcentaje sobre el total de la población)

$<2 \% \quad 2-4 \% \quad 4-6 \% \quad 6-8 \% \quad \geq 8 \%$

Fuente: elaboración propia a partir de los datos del Padrón Municipal de Habitantes

En cuanto a las variaciones en los porcentajes (Figura 9), entre 2002 y 2007 las áreas urbanas que más se sobrenvejecieron fueron las de Asturias, Palencia o Bilbao con un incremento algo superior al $20 \%$, dato que contrasta con la disminución, siempre por debajo del 10 \%, detectada en Tenerife Sur, Arrecife, Costa del Sol, Roquetas, Denia-Jávea y Aranjuez. Entre 2007 y 2012 no hay ni un solo caso de disminución; por el contrario, se producen importantes incrementos de la proporción de la población de 80 y más años en algunas de las áreas que acabamos de citar como Tenerife Sur o Denia-lávea, a las que se suman Gran Canaria y Orihuela (con incrementos de entre $25-27 \%$ ) y el llamativo caso de Torrevieja, que en este quinquenio ve aumentar la proporción de personas de 80 y más años en un 38 \%. ¿Se deben estos crecimientos a desajustes en las cifras padronales? Quizá estas personas, aun residiendo desde hace tiempo en las áreas urbanas señaladas, han formalizado su registro en el padrón solo al necesitar de atenciones o servicios públicos por razón de su edad, por lo que estos incrementos tan considerables están, en realidad, haciendo aflorar una realidad. En cualquier caso, somos conscientes de las limitaciones de la fuente a la hora de considerar a los extranjeros residentes en España (Rodríguez Rodríguez \& Casado-Díaz, 2002; Rodríguez, Lardiés, \& Rodríguez, 
2010). Los contrastes se vuelven a atenuar entre 2012 y 2017, cuando las variaciones más altas se sitúan ligeramente por encima del 20 \% en áreas urbanas como Gran Canaria, Las Palmas y, en la Península, en Ponferrada, Talavera de la Reina o Blanes-Lloret. En este quinquenio, Costa Blanca y Denia-Jávea vuelven a mostrar una ligera disminución de las personas de 80 y más años.

Figura 9. Variación del porcentaje de personas de 80 y más años en las áreas urbanas españolas
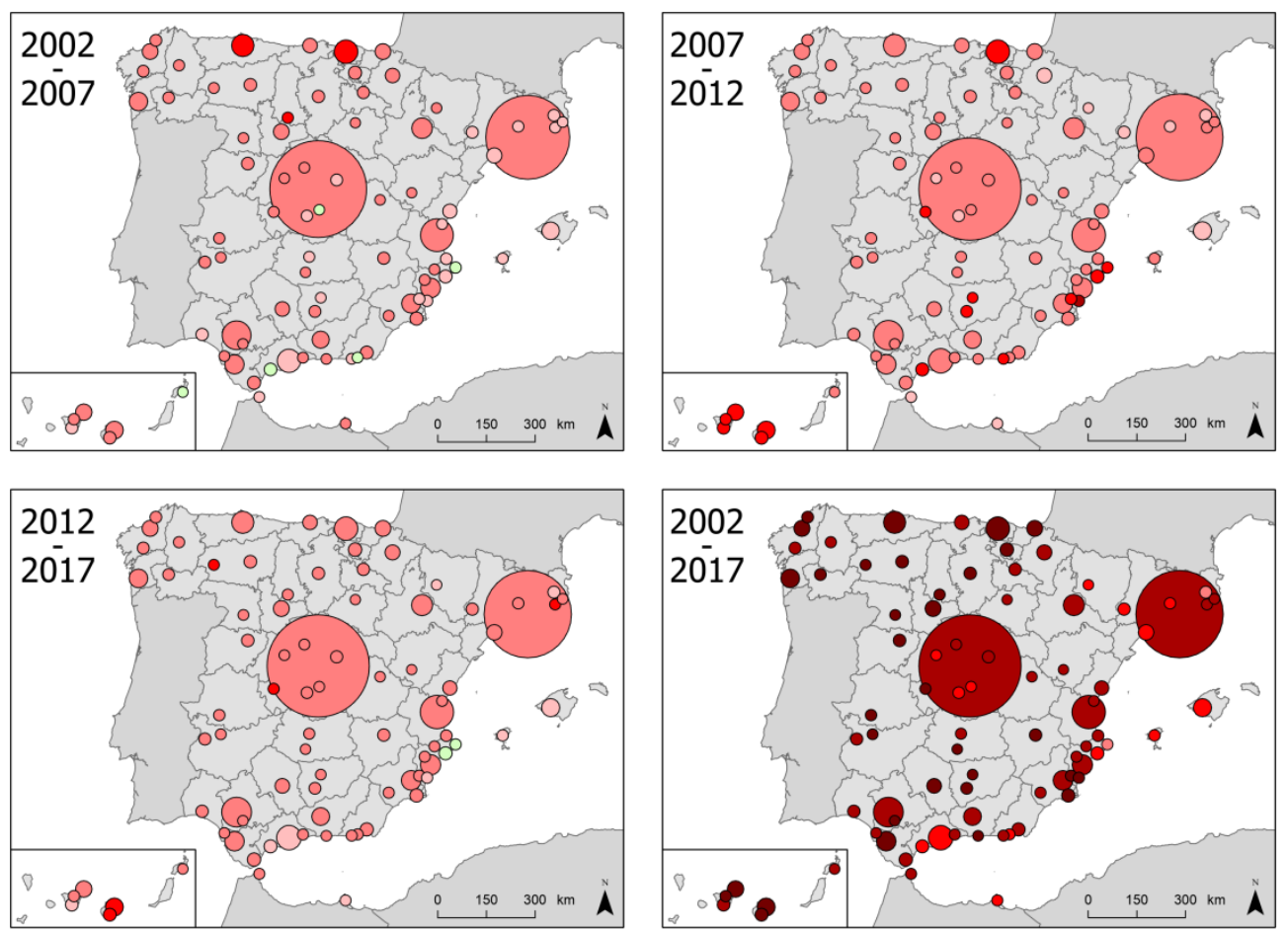

Volumen de población (en miles de habitantes)
Variación de la población de 80 y más años (porcentaje de cambio en el período indicado)

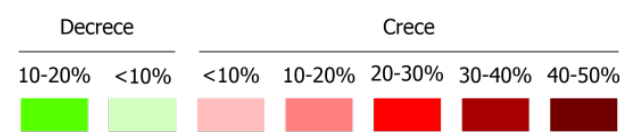

Fuente: elaboración propia a partir de los datos del Padrón Municipal de Habitantes

En el conjunto del período, el mapa que compara 2002 con 2017 refleja un avance claro y significativo del sobrenvejecimiento de las áreas urbanas. Así, 33 de ellas han visto aumentar el porcentaje de esta población entre un $40 \%$ y un $50 \%$; 38, entre un $30 \%$ y un $40 \%$; 13, entre un $20 \%$ y un 30 \%; y solo 2, Denia-ávea y Girona, entre un 10 \% y un 20 \%. No hay ningún caso de área urbana con un crecimiento inferior al $10 \%$ o que haya visto disminuir el sobrenvejecimiento. 


\section{Incidiendo en las relaciones entre el envejecimiento y el sobrenvejecimiento urbanos}

Es conocida la relación lógica entre el envejecimiento y el sobrenvejecimiento en las áreas urbanas (Pons y Montoro, 2020). La figura 10 cruza los porcentajes de población mayor de 65 y más años con los de población mayor de 80 y más años por áreas urbanas españolas, representadas por puntos en el gráfico de dispersión. El resultado de este cruce refleja con claridad una tendencia lineal. En otras palabras, las áreas urbanas con menor tasa de envejecimiento son también las áreas urbanas con menor sobrenvejecimiento (Gran Canaria Sur, Tenerife Sur, Arrecife, El Ejido, Roquetas de Mar, Melilla, Ceuta, etc.) y las áreas urbanas con mayor tasa de envejecimiento son las que presentan los mayores niveles de sobrenvejecimiento (Ourense, León, Zamora, Ferrol, Asturias, Ponferrada, Salamanca, etc.).

Figura 10. Relación entre envejecimiento y sobrenvejecimiento de las áreas urbanas (2017)

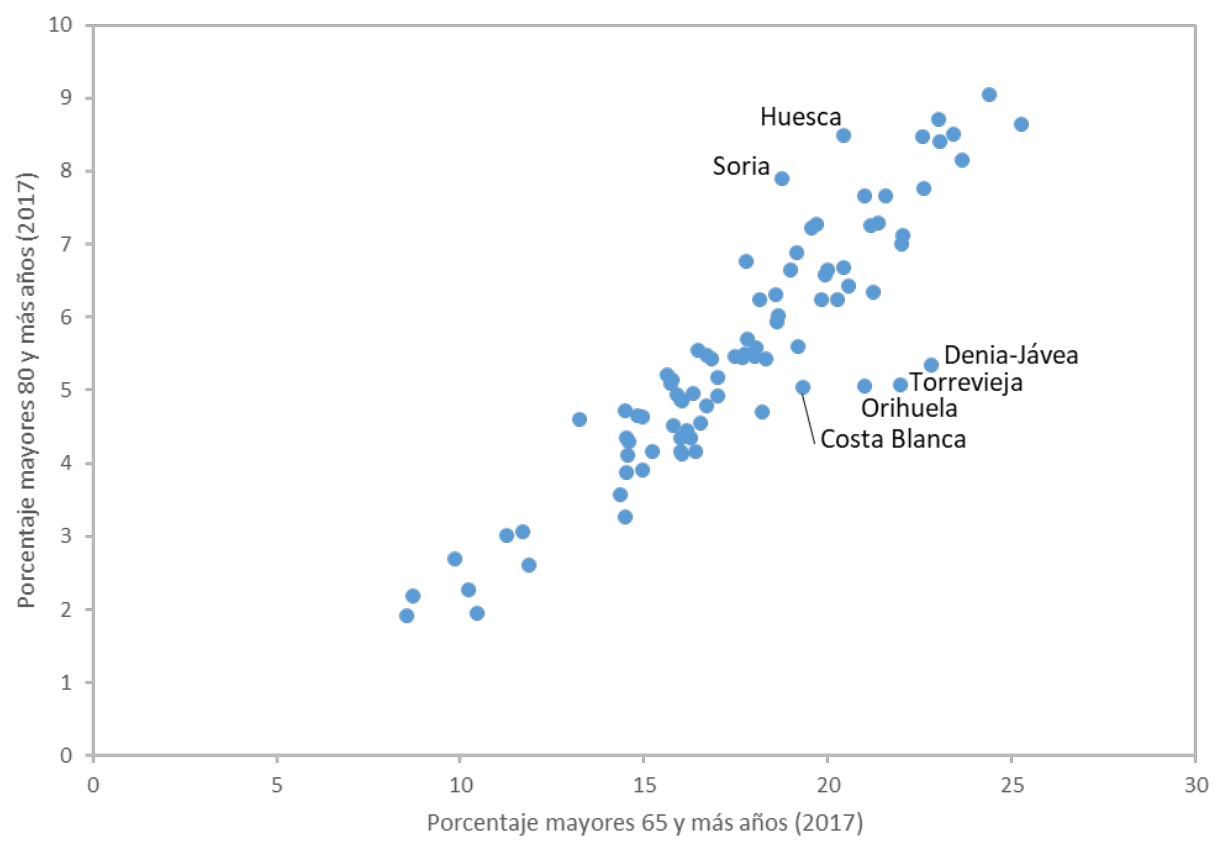

Fuente: elaboración propia a partir de los datos del Padrón Municipal de Habitantes

A pesar de que el resultado pueda parecer excesivamente obvio, realizar este cruce permite identificar las áreas urbanas cuyo nivel de sobrenvejecimiento es más acusado que el que les correspondería de acuerdo con el nivel de envejecimiento que tienen -aquellas que aparecen por encima de la línea, notablemente los casos de Huesca y Soria- y, también, las que presentan un nivel de sobrenvejecimiento inferior respecto a su propio nivel de envejecimiento 
- las que aparecen por debajo de la línea-. En esta última situación se encuentran las áreas urbanas de Denia-Jávea, Torrevieja, Orihuela o Cosła Blanca.

Teniendo esta relación como telón de fondo, ¿cuáles han sido las dinámicas del envejecimiento y del sobrenvejecimiento demográfico en el período que ahora se analiza?, ¿cómo quedan retratadas las áreas urbanas españolas?

\subsection{Modelos de envejecimiento y sobrenvejecimiento}

Se ha realizado una tipología o modelización de las áreas urbanas de 4 categorías combinando el nivel inicial de envejecimiento (o sobrenvejecimiento) con la intensidad del cambio en el período estudiado. En el caso del envejecimiento, la tasa media de las áreas urbanas en 2002 (igual a 15,4 \%) y la variación porcentual media de envejecimiento demográfico en el período 2002 a 2017 (igual a 14 \%) son los valores empleados para delimitar las categorías. El resultado se muestra en un gráfico de dispersión (Figura 11). Las áreas urbanas están representadas por puntos e identificadas por un código numérico. Su ubicación en uno de los cuadrantes evidencia modelos de envejecimiento diferentes.

En el cuadrante 1 están las áreas urbanas cuya situación con respecto al envejecimiento demográfico es más favorable. Partían de tasas bajas de envejecimiento en 2002 y además el ritmo o intensidad ha sido inferior a la media. Se trata de un total de 18 áreas, cinco de ellas del interior de la Península (Mérida, Guadalajara, Toledo, Ciudad Real, Jaén), las dos de Baleares (Eivissa y Palma), nueve más distribuidas por la costa mediterránea (Girona, Tarragona-Reus, Castellón de la Plana, Murcia, Cartagena, Lorca, Almería, El Ejido, Granada) y las ciudades autónomas (Ceuta y Melilla).

En contraposición, en el cuadrante 4 aparecen las 16 áreas urbanas que, partiendo de una tasa alta de envejecimiento, en estos quince años han experimentado un proceso de envejecimiento superior a la media. En otras palabras, se trata de las áreas urbanas en situación más desfavorable. Entre ellas se encuentran cuatro de las seis gallegas (Vigo-Pontevedra, A Coruña, Ferrol, Ourense), todas las de la costa cantábrica (Asturias y Santander-Torrelavega en el límite, y claramente Bilbao y Donostia/San Sebastián), gran parte de las áreas urbanas de Castilla-León (Zamora, Salamanca, Valladolid, Palencia, Burgos), Orihuela, Valencia y Sant Feliú de Guixols. 


\section{Figura 11. Modelos de envejecimiento demográfico de las áreas urbanas}

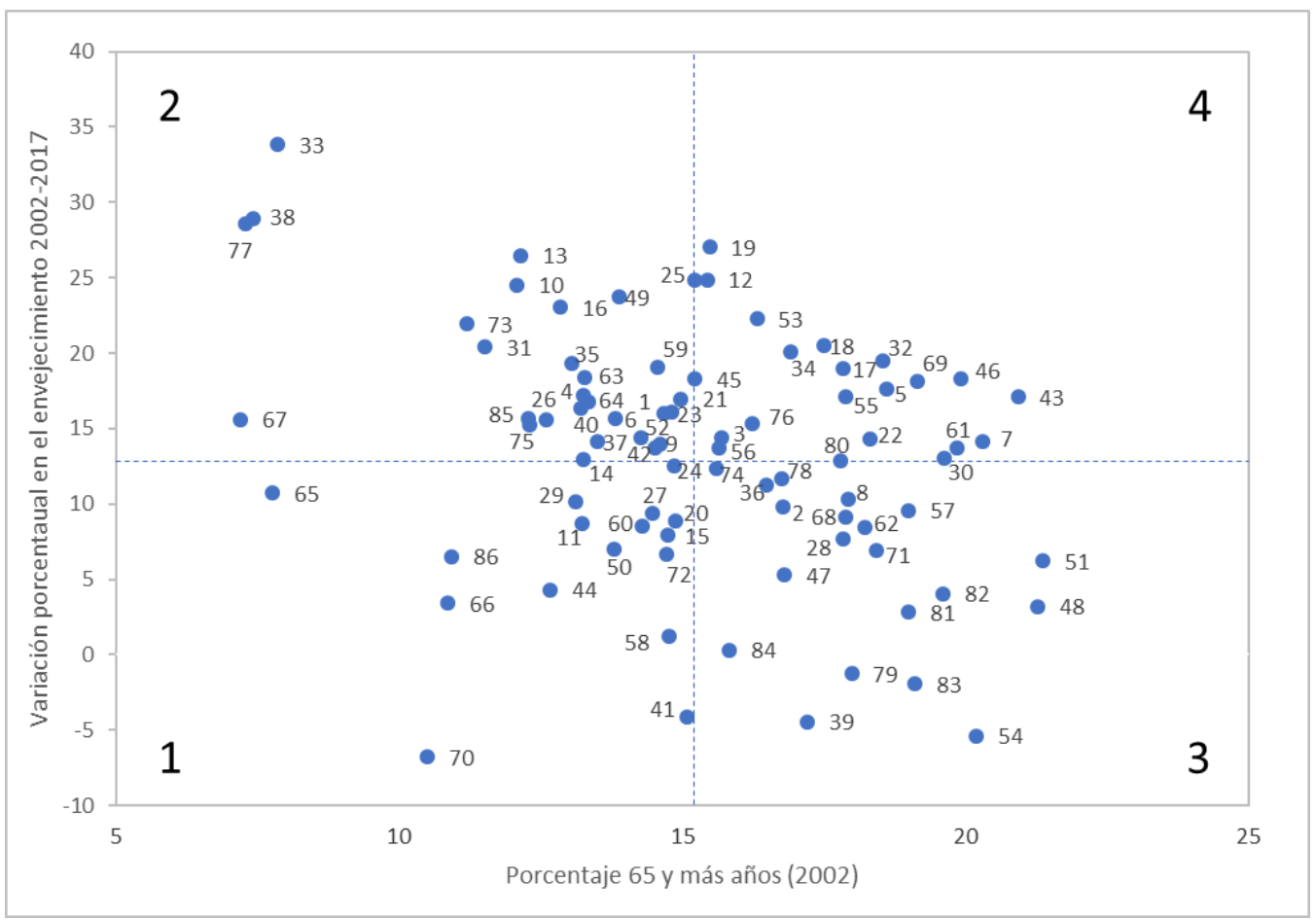

Leyenda: 1: Madrid; 2: Barcelona; 3: Valencia; 4: Sevilla; 5: Bilbao; 6: Málaga; 7: Asturias; 8: Zaragoza; 9: Alicante-Elche; 10: Bahía de Cádiz; 11: Murcia; 12: Vigo-Pontevedra; 13: Las Palmas de Gran Canaria; 14: Palma; 15: Granada; 16: Santa Cruz de Tenerife-La Laguna; 17: A Coruña; 18: Donostia/San Sebastián; 19: Valladolid; 20 : Tarragona-Reus; 21: Córdoba; 22: Santander-Torrelavega; 23: Pamplona; 24: Castellón de la Plana; 25: Vitoria/Gasteiz; 26: Bahía de Algeciras; 27: Cartagena; 28: Costa Blanca; 29: Almería; 30: León; 31: Costa del Sol; 32: Salamanca; 33: Gran Canaria Sur; 34: Burgos; 35: Huelva; 36: Logroño; 37: Albacete; 38: Tenerife Sur; 39: Lleida; 40: Badajoz; 41: Girona; 42: Jaén; 43: Ferrol; 44: Guadalajara; 45: Santiago de Compostela; 46: Ourense; 47: Gandía; 48: Torrevieja; 49: Valle de la Orotava; 50: Toledo; 51: Denia-ávea; 52: Cáceres; 53: Orihuela; 54: Manresa; 55: Palencia; 56: Talavera de la Reina; 57: Lugo; 58: Lorca; 59: Elda-Petrer; 60: Ciudad Real; 61: Ponferrada; 62: Alcoy/Alcoi; 63: Blanes-Lloret de Mar; 64: Vélez-Málaga; 65: El Ejido; 66: Ceuta; 67: Roquetas de Mar; 68: Segovia; 69: Zamora; 70: Melilla; 71: Sagunto/Sagunt; 72: Mérida; 73: Sanlúcar de Barrameda; 74: Linares; 75: Motril; 76: Sant Feliú de Guixols; 77: Arrecife; 78: Ávila; 79: Cuenca; 80: Puertollano; 81: Huesca; 82: Soria; 83: Teruel; 84: Aranjuez; 85: Utrera; 86: Eivissa.

Fuente: elaboración propia a partir de los datos del Padrón Municipal de Habitantes

En el cuadrante 3 se sitúan 24 áreas urbanas que, partiendo como las anteriores de una tasa alta de envejecimiento, sin embargo, en este período han tenido un proceso de envejecimiento más moderado que la media. Se trata de áreas urbanas del interior (Lugo, Ponferrada, León, Ávila, Segovia, Aranjuez, Talavera de la Reina, Soria, Logroño, Huesca, Zaragoza, Teruel, Cuenca, Lleida; más Puertollano y Linares más al sur) y de la costa mediterránea en la Península (Manresa, Barcelona, Sagunto/Sagunt, Gandía, Denia-jávea, Alcoy/Alcoi, Costa Blanca, Torrevieja). 
Finalmente, en el cuadrante 2 figuran 28 áreas urbanas que, partiendo de unas tasas de envejecimiento bajas al comienzo del período, han experimentado un cambio acusado y su ritmo de envejecimiento demográfico puede definirse como intenso. En este grupo están todas las áreas urbanas de las Islas Canarias (Tenerife Sur, Valle de la Orotava, Santa Cruz de Tenerife-La Laguna, Gran Canaria Sur, Las Palmas de Gran Canaria, Arrecife), una comunidad calificada demográficamente hasta hace poco como 'joven' en el contexto nacional, que afronta un proceso de envejecimiento acentuado por razón de unas voluminosas generaciones del baby boom, una natalidad y fecundidad muy bajas y, unas tasas migratorias internacionales escasas e inter-autonómicas negativas (Díaz Hernández \& Domínguez Mújica, 2014). También están incluidas la gran mayoría de las áreas urbanas andaluzas (Huelva, Sevilla, Sanlúcar de Barrameda, Utrera, Bahía de Cádiz, Bahía de Algeciras, Costa del Sol, Málaga, Vélez-Málaga, Motril, Roquetas de Mar y ya en el interior, Córdoba y hasta seis áreas más del interior peninsular, incluyendo Cáceres (en el límite con el cuadrante 3), Badajoz, Albacete, Madrid, Vitoria/Gasteiz y Pamplona, y otras cuatro en la costa: Alicante-Elche (en el límite con el cuadrante 3) y Elda-Petrer en la provincia de Alicante, Blanes-Lloret de Mar y Santiago de Compostela.

Centrando nuestro análisis en la dinámica del sobrenvejecimiento demográfico, repetimos el gráfico de dispersión con el eje ' $x$ ' seccionado por la tasa media de sobrenvejecimiento de las áreas urbanas en 2002 (igual a 3,5 \%) y con el eje 'y' seccionado por la variación porcentual media del sobrenvejecimiento entre 2002 y 2017 (igual a 36,1 \%) (Figura12). De estas cifras se desprende un hecho obvio por los grupos de edad que entran en el cálculo de ambas tasas, como es que la tasa de sobrenvejecimiento está claramente por debajo de la tasa de envejecimiento; y, también, que la variación media del sobrenvejecimiento es muy superior (más del doble) a la variación media del envejecimiento. 
Figura 12. Modelos de sobrenvejecimiento demográfico de las áreas urbanas

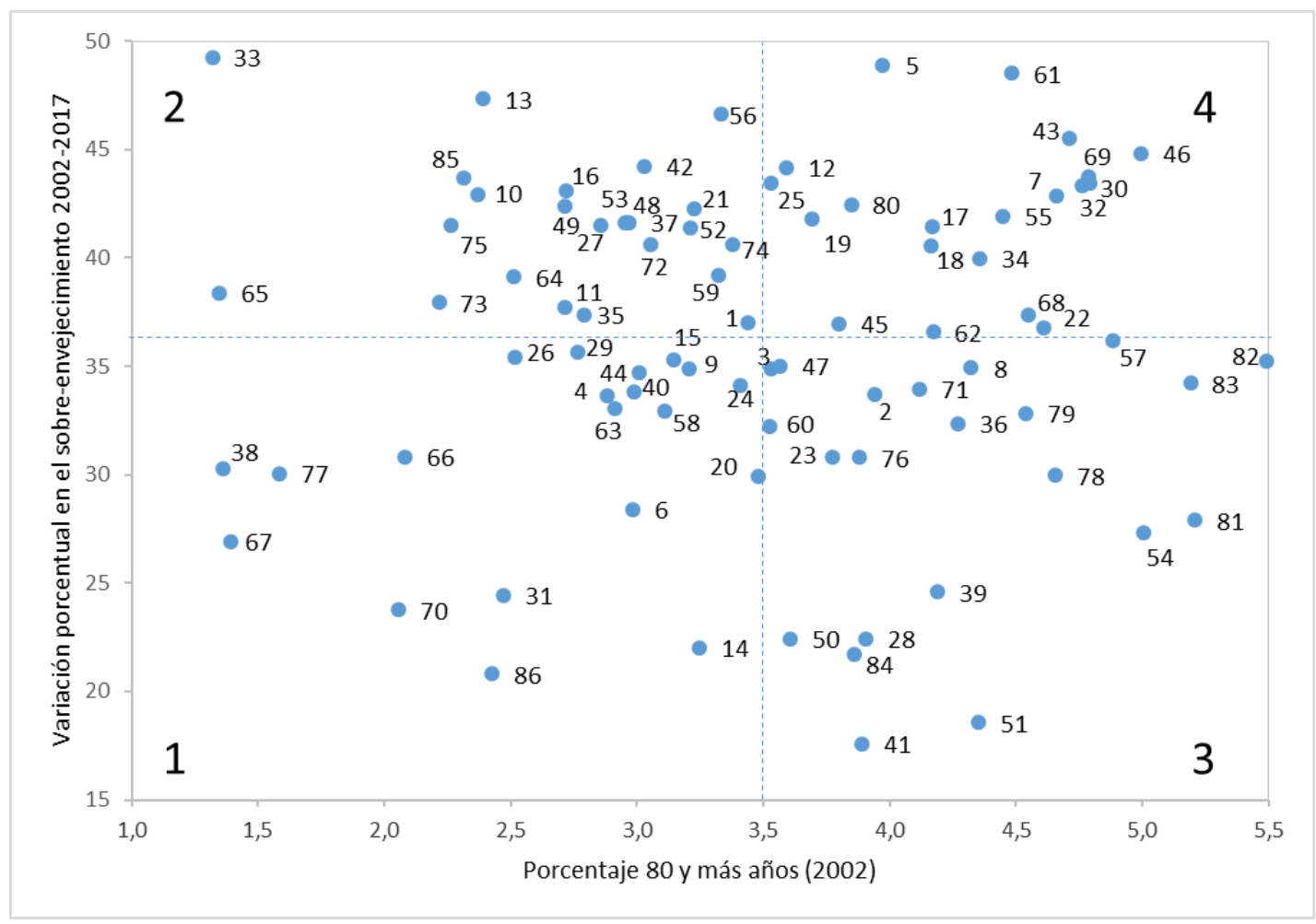

Leyenda: 1: Madrid; 2: Barcelona; 3: Valencia; 4: Sevilla; 5: Bilbao; 6: Málaga; 7: Asturias; 8: Zaragoza; 9: Alicante-Elche; 10: Bahía de Cádiz; 11: Murcia; 12: Vigo-Pontevedra; 13: Las Palmas de Gran Canaria; 14: Palma; 15: Granada; 16: Santa Cruz de Tenerife-La Laguna; 17: A Coruña; 18: Donostia/San Sebastián; 19: Valladolid; 20: Tarragona-Reus; 21: Córdoba; 22: Santander-Torrelavega; 23: Pamplona; 24: Castellón de la Plana; 25 : Vitoria/Gasteiz; 26: Bahía de Algeciras; 27: Cartagena; 28: Costa Blanca; 29: Almería; 30: León; 31: Costa del Sol; 32: Salamanca; 33: Gran Canaria Sur; 34: Burgos; 35: Huelva; 36: Logroño; 37: Albacete; 38: Tenerife Sur; 39: Lleida; 40: Badajoz; 41: Girona; 42: Jaén; 43: Ferrol; 44: Guadalajara; 45: Santiago de Compostela; 46: Ourense; 47: Gandía; 48: Torrevieja; 49: Valle de la Orotava; 50: Toledo; 51: Denia-Jávea; 52: Cáceres; 53: Orihuela; 54: Manresa; 55: Palencia; 56: Talavera de la Reina; 57: Lugo; 58: Lorca; 59: Elda-Petrer; 60: Ciudad Real; 61: Ponferrada; 62: Alcoy/Alcoi; 63: Blanes-Lloret de Mar; 64: Vélez-Málaga; 65: El Ejido; 66: Ceuta; 67: Roquetas de Mar; 68: Segovia; 69: Zamora; 70: Melilla; 71: Sagunto/Sagunt; 72: Mérida; 73: Sanlúcar de Barrameda; 74: Linares; 75: Motril; 76: Sant Feliú de Guixols; 77: Arrecife; 78: Ávila; 79: Cuenca; 80: Puertollano; 81: Huesca; 82: Soria; 83: Teruel; 84: Aranjuez; 85: Utrera; 86: Eivissa.

Fuente: elaboración propia a partir de los datos del Padrón Municipal de Habitantes

\subsection{Comparación de los modelos de envejecimiento y sobrenvejecimiento}

La comparación de las Figuras 11 y 12 permite afinar en la evolución de la relación entre el envejecimiento y el sobrenvejecimiento de las áreas urbanas. En la Figura 12 encontramos que el número de áreas urbanas está muy repartido por los cuadrantes; hay 20 áreas urbanas en el cuadrante 1, 21 en los cuadrantes 3 y 4, y 24 áreas urbanas en el cuadrante 2. Sin embargo, 
esta distribución más "igualitaria" puede leerse en clave de una mayor polarización de los resultados de sobrenvejecimiento en comparación con los de envejecimiento: los cuadrantes que han aumentado el número de áreas urbanas son el 1 (en 2 áreas), que refleja la situación más positiva con respecto al envejecimiento y el 4 (en 4 áreas), que refleja la situación más negativa al respecto. Si nos fijamos en las áreas urbanas en sí, las gráficas de dispersión de ambas dinámicas muestran, en el caso del cuadrante 1, una coincidencia del $50 \%$ de las áreas urbanas; este porcentaje de coincidencia se eleva al $62 \%$ y $63 \%$ en el caso de los cuadrantes 4 y 2 y a un $70 \%$ en el cuadrante 3. En otras palabras, las diferencias en las variaciones porcentuales, aun reflejando la relación entre los dos envejecimientos en las áreas urbanas, permiten detectar aquellos casos en los que estas dos realidades no están totalmente "en línea", en los que un área urbana está caracterizada en un cuadrante desde el punto de vista de la dinámica del envejecimiento y en otro desde la del sobrenvejecimiento.

Un total de 33 áreas urbanas reflejan alguna disonancia. En 15 áreas urbanas los dos niveles de envejecimiento eran bajos en 2002 pero, en 10 casos la intensidad de envejecimiento entre 2002 y 2017 ha sido alta (cuadrante 2), mientras que la de sobrenvejecimiento ha sido baja (cuadrante 1). Se trata de Badajoz, Sevilla, Bahía de Algeciras, Costa del Sol, Málaga, Roquetas, Alicante, Blanes-Lloret de Mar, Tenerife Sur y Arrecife. En los otros 5 casos -Murcia, Cartagena, Jaén, El Ejido, Mérida- sucede al contrario: la intensidad del envejecimiento ha sido baja (cuadrante 1) mientras que la del sobrenvejecimiento ha sido alta (cuadrante 2). Por el contrario, en 8 áreas urbanas los dos niveles de envejecimiento eran altos en 2002 pero, en 2 casos la intensidad de envejecimiento entre 2002 y 2017 ha sido alta (cuadrante 4), mientras que la de sobrenvejecimiento ha sido baja (cuadrante 3). Se trata de Sant Feliú de Guixols y Valencia. En los otros 6 casos -Lugo, Ponferrada, León, Segovia, Puertollano, Alcoy/Alcoi- sucede al contrario: la intensidad del envejecimiento ha sido baja (cuadrante 3) mientras que la del sobrenvejecimiento ha sido alta (cuadrante 4).

Dos áreas urbanas se caracterizan por intensidades de envejecimiento y sobrenvejecimiento altas, pero con una tasa de envejecimiento baja (cuadrante 2) y una tasa de sobrenvejecimiento alta (cuadrante 4): Santiago de Compostela y Vitoria-Gasteiz. El caso de Orihuela, que comparte con ellas intensidades altas de variación en los dos indicadores, destaca por el contrario, una tasa de envejecimiento alta y una de sobrenvejecimiento baja. Tres áreas urbanas coinciden en intensidades bajas de variación en los dos indicadores y en unos niveles de envejecimiento débiles (cuadrante 1) frente a unos niveles de sobrenvejecimiento altos (cuadrante 3) en 2002: Toledo, Ciudad Real, y Girona. 
Finalmente, las áreas urbanas de Talavera de la Reina, Linares y Torrevieja estaban situadas en el cuadrante 3 con respecto al envejecimiento (tasas altas en 2002 y variación porcentual baja 2002-2017) y en el cuadrante 2 en cuanto a sobrenvejecimiento (tasas bajas en 2002 y variación porcentual baja 2002-2017), y el área urbana de Pamplona, justo al revés: cuadrante 2 en envejecimiento y cuadrante 3 en sobrenvejecimiento.

La figura 13 permite visualizar geográficamente esta comparación. A grandes trazos, la gran mayoría de las áreas urbanas de las regiones ubicadas en el sector noroccidental de España, especialmente en el caso del sobrenvejecimiento, pertenecen a la categoría más negativa del modelo planteado; el resto del norte e interior español tiene unas áreas urbanas clasificadas en la categoría siguiente, que se ha beneficiado de una intensidad de cambio más benévola. Las áreas urbanas ubicadas más al sur, incluido el litoral mediterráneo, presentan una situación mejor a pesar de una intensidad de cambio elevada gracias a partir de niveles bajos. Y, finalmente, las áreas urbanas ubicadas en la categoría más positiva, "salpimentan" el interior de la meseta sur y algunos puntos de la costa mediterránea -en el caso del envejecimiento- y las áreas urbanas del litoral andaluz en el caso del sobrenvejecimiento.

Figura 13. Modelos de envejecimiento y sobrenvejecimiento urbano (2002-2017)
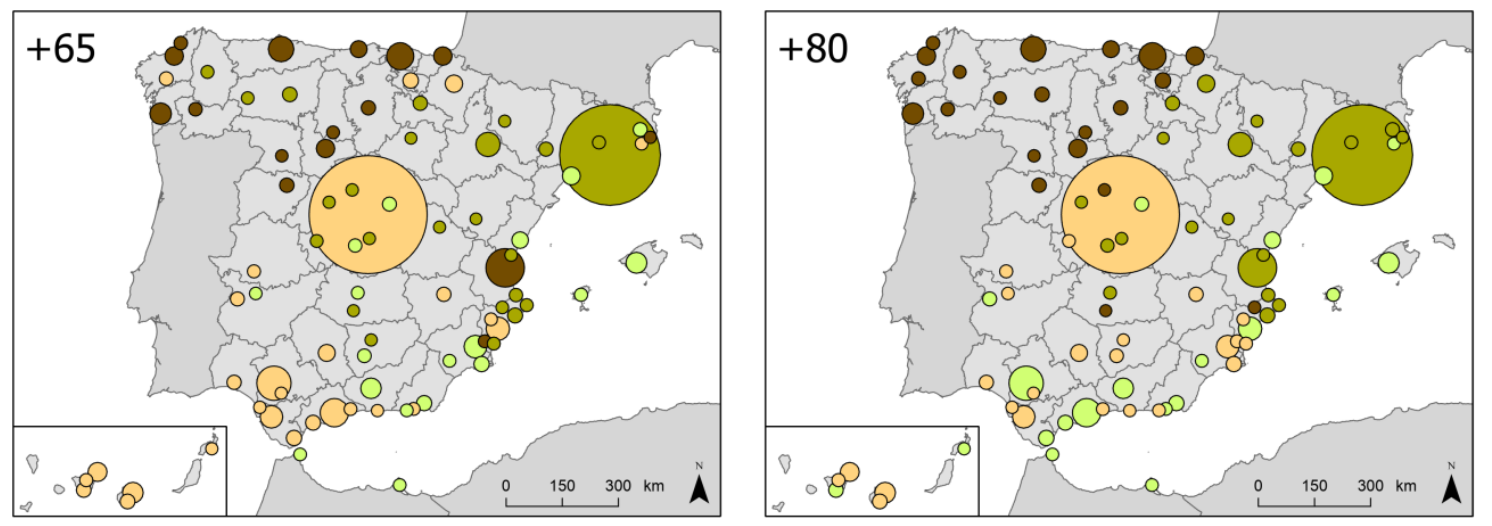

Volumen de población (en miles de habitantes)

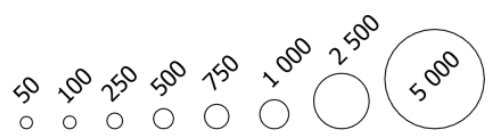

\section{Modelos de envejecimiento}

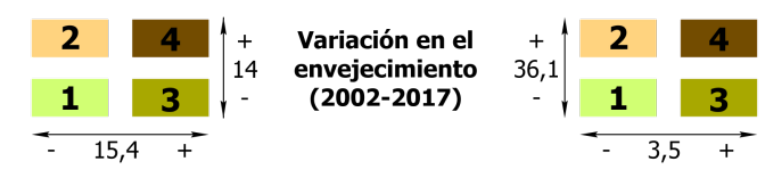

Porcentaje de población de 65 y más años (2002)
Porcentaje de población de 80 y más años (2002)

Fuente: elaboración propia a partir de los datos del Padrón Municipal de Habitantes 


\subsection{Estructura de la población de 60 y más años de las áreas urbanas según modelo de envejecimiento}

Tal como hacíamos en el punto 3 al analizar los contrastes en el envejecimiento entre el mundo rural y el mundo urbano en España en 2017, ahora volvemos a utilizar los indicadores de edad media de la población y las pirámides de edad de personas de 60 y más años para matizar los contrastes existentes en el perfil de la población urbana en su conjunto con el de la población urbana residente en alguno de los 4 tipos de áreas urbanas según su modelo de envejecimiento. La edad media de la población urbana española en 2017 es de 42,4 años, pero esta cifra oscila de forma significativa de los 40,2 años de media en las áreas urbanas englobadas en el modelo 1 de envejecimiento a los 41,6 años en las del modelo 2, los 42,9 años en las del modelo 3 y, finalmente, a los 44,8 años en las áreas urbanas del modelo 4.

En cuanto a las pirámides de personas de 60 y más años, los contrastes más acusados se encuentran en los modelos 1 y 4 (Figura 14). Recordemos que el modelo 1 recoge la situación más benévola con respecto al envejecimiento demográfico, lo que queda bien patente en la pirámide de la población: prácticamente todas las cohortes del modelo 1 tienen menos peso porcentual (de forma más marcada las femeninas) sobre el conjunto de la población que el perfil urbano general. El modelo 4, por el contrario, que representa a todas las áreas urbanas con la situación menos favorable con respecto al envejecimiento demográfico, tiene una pirámide cuyas cohortes de adultos mayores, tanto masculinas como femeninas, sobrepasan a las urbanas en su conjunto. Los modelos 2 y 3, que reflejan situaciones intermedias -caracterizadas por ser opuestas: de bajo envejecimiento y alta intensidad de cambio o de alto envejecimiento y baja intensidad de cambio - tienen una estructura por edades y sexos sensiblemente más parecida a la del conjunto de la población urbana.

La tasa de feminidad de estas poblaciones aporta otro matiz interesante a la comparación. Entre los de 65 y más años el valor más bajo lo encontramos, como cabía esperar, en las áreas urbanas del modelo 1, con 1,33 mujeres por cada hombre. De hecho, las cifras de las áreas urbanas del modelo 2 -con 1,37 mujeres por cada hombre- y del modelo 3 -con 1,36siguen estando por debajo de la media urbana española -igual a 1,38-. Son las áreas urbanas enmarcadas en el modelo 4 las que elevan la media hasta 1,41 mujeres por cada hombre de 65 y más años. Entre la población de 80 y más años la tasa de feminidad se eleva a 1,76 en el caso del modelo 1, seguido de 1,8 en el modelo 3, 1,84 en el 2 y, 1,9 mujeres por cada hombre en el modelo 4, frente a la media urbana española de 1,83 mujeres por cada hombre de 80 y más 
años. Estos datos parecen apuntar a que un ritmo más fuerte de variación del envejecimiento en el período analizado (modelo 2) tiene mayores repercusiones que partir de unos niveles de envejecimiento más elevados (modelo 3) en la tasa de feminidad de las personas de 80 y más años.

Figura 14. Perfil de las personas de 60 y más años por edad, sexo y entorno de residencia en España (2017)
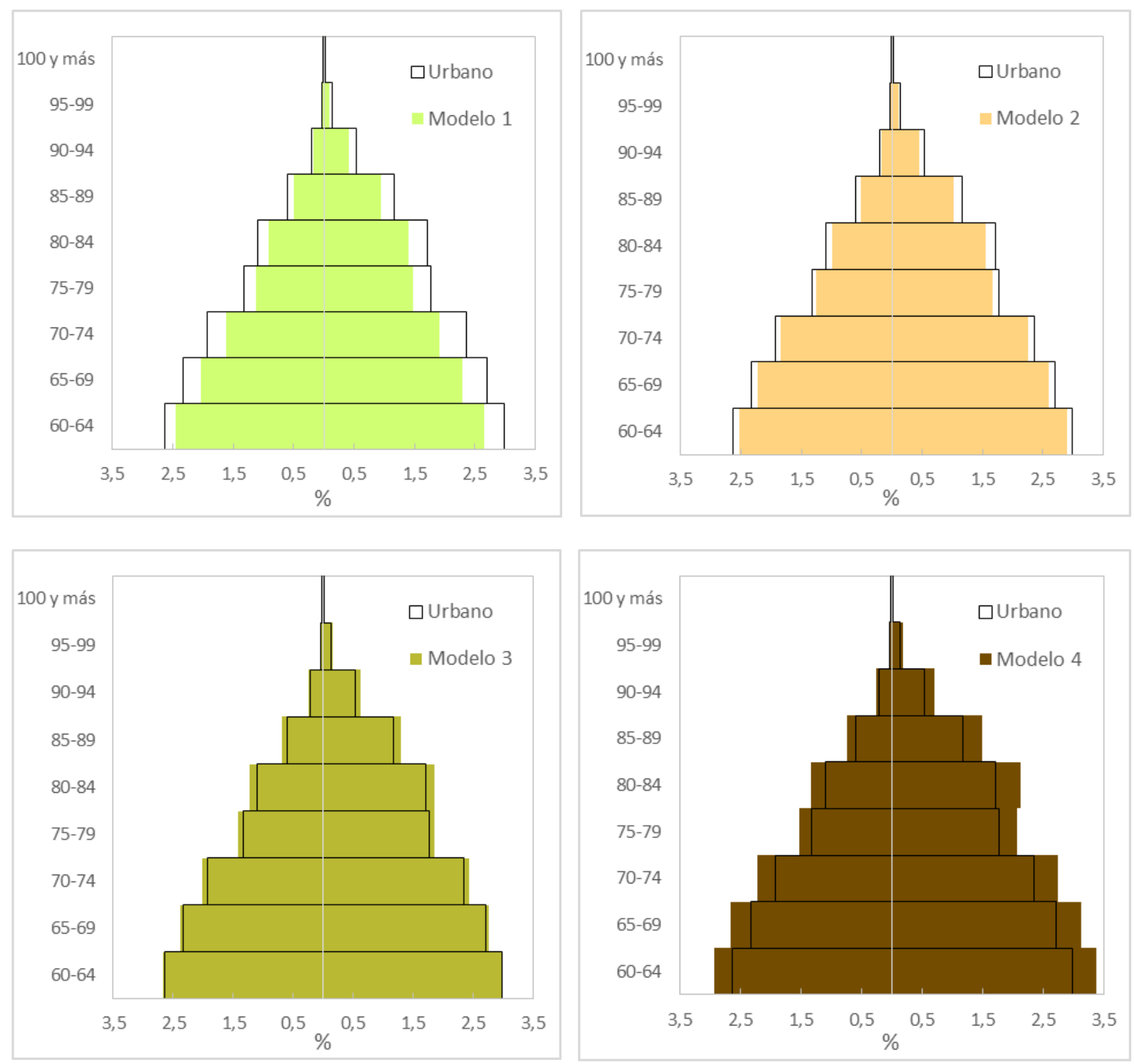

Fuente: elaboración propia a partir de los datos del Padrón Municipal de Habitantes

\subsection{Relación entre modelos de envejecimiento y sobrenvejecimiento y la población extranjera}

Responder a la pregunta de qué efectos ha tenido la inmigración en el proceso de envejecimiento de las áreas urbanas no es tarea fácil. El Atlas Estadístico de las Áreas Urbanas 
en España recoge información procedente del Padrón y facilita el volumen de población extranjera total y por continente de origen en las áreas urbanas. En este análisis se va a emplear a la población extranjera como proxy de población inmigrante para explorar las relaciones con el envejecimiento y el sobrenvejecimiento. Se asume, así, que las cifras empleadas son una parte de la realidad, mermada por los procesos de naturalización de personas inmigrantes extranjeras (que pueden seguir residiendo en territorio español) y, por el hecho de obviar el alcance de la inmigración no extranjera.

La figura 15 presenta la evolución de la población extranjera en España entre 2002 y 2017 en volúmenes y porcentaje sobre el total de población en las áreas urbanas.

Figura 15. Volumen y proporción de población de nacionalidad extranjera de las áreas urbanas españolas (2002-2017)
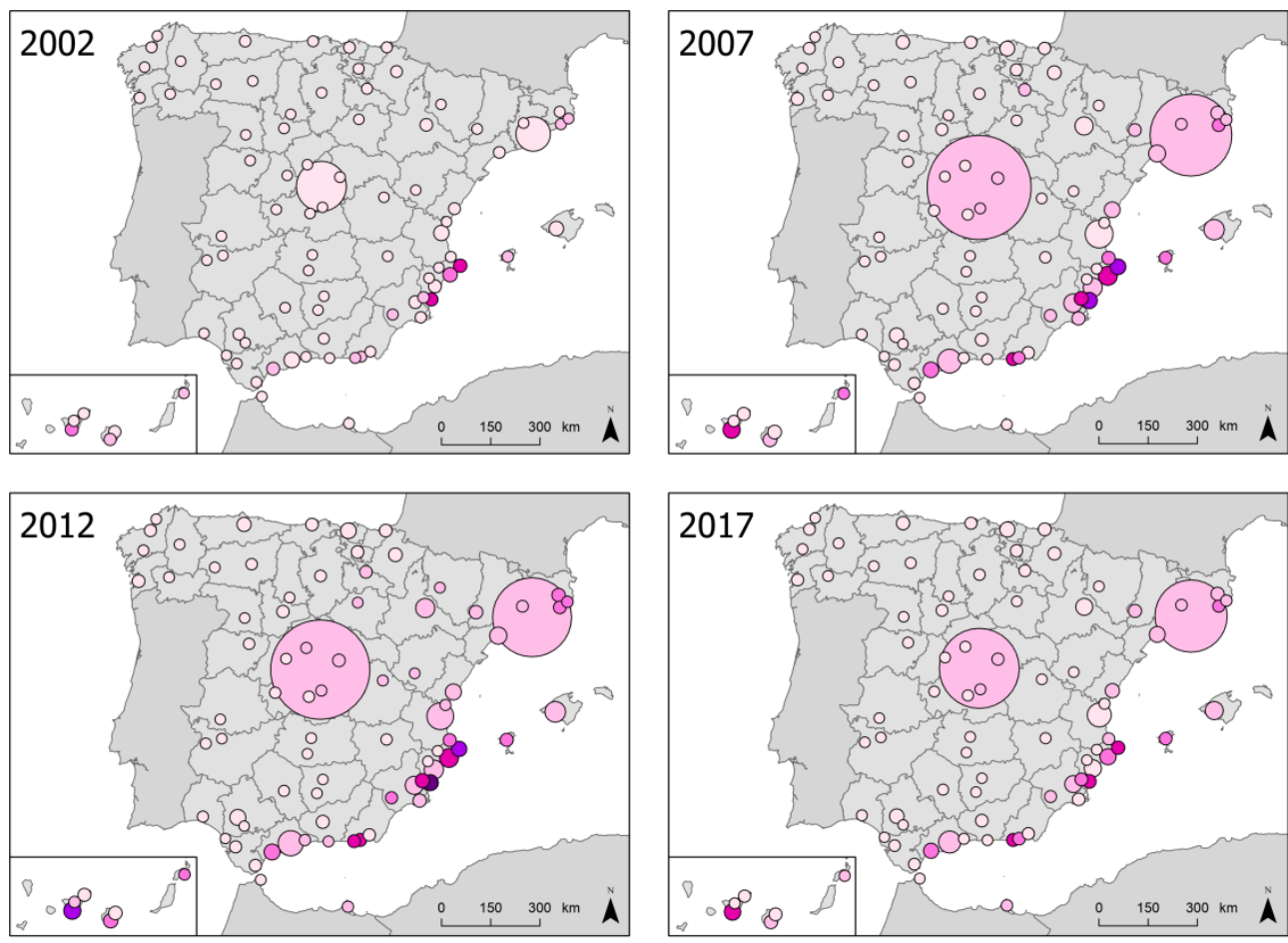

Volumen de población extranjera (en miles de habitantes)

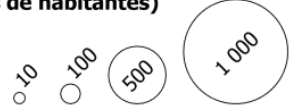

Porcentaje de población extranjera

(sobre el total de la población)

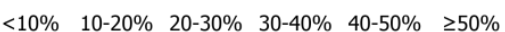

Fuente: elaboración propia a partir de los datos

del Atlas Estadístico de las Áreas Urbanas en España

Se han calculado los coeficientes de correlación con el fin de medir la fuerza y el sentido de la relación entre el envejecimiento y la presencia de población extranjera en los años estudiados. Recordemos que, cuanto más lejos esté el valor del coeficiente de cero -en positivo o en 
negativo-, más importancia tiene la relación entre estas dos variables. La figura 16 muestra los coeficientes de la tasa de envejecimiento y el porcentaje de población extranjera para el conjunto de las áreas urbanas del país y para los cuatro grupos de áreas urbanas según el modelo envejecimiento. Además, partiendo del hecho de ser España un país de referencia como destino de la movilidad europea de personas retiradas (Durán Muñoz, 2016; García Jiménez \& Schriewer, 2009; Rodríguez et al., 2005), se consideró de interés calcular los coeficientes de correlación entre el envejecimiento y esta población extranjera en particular.

Figura 16. Coeficientes de correlación tasas envejecimiento (65 y más años) y población extranjera de las áreas urbanas españolas (2002-2017)
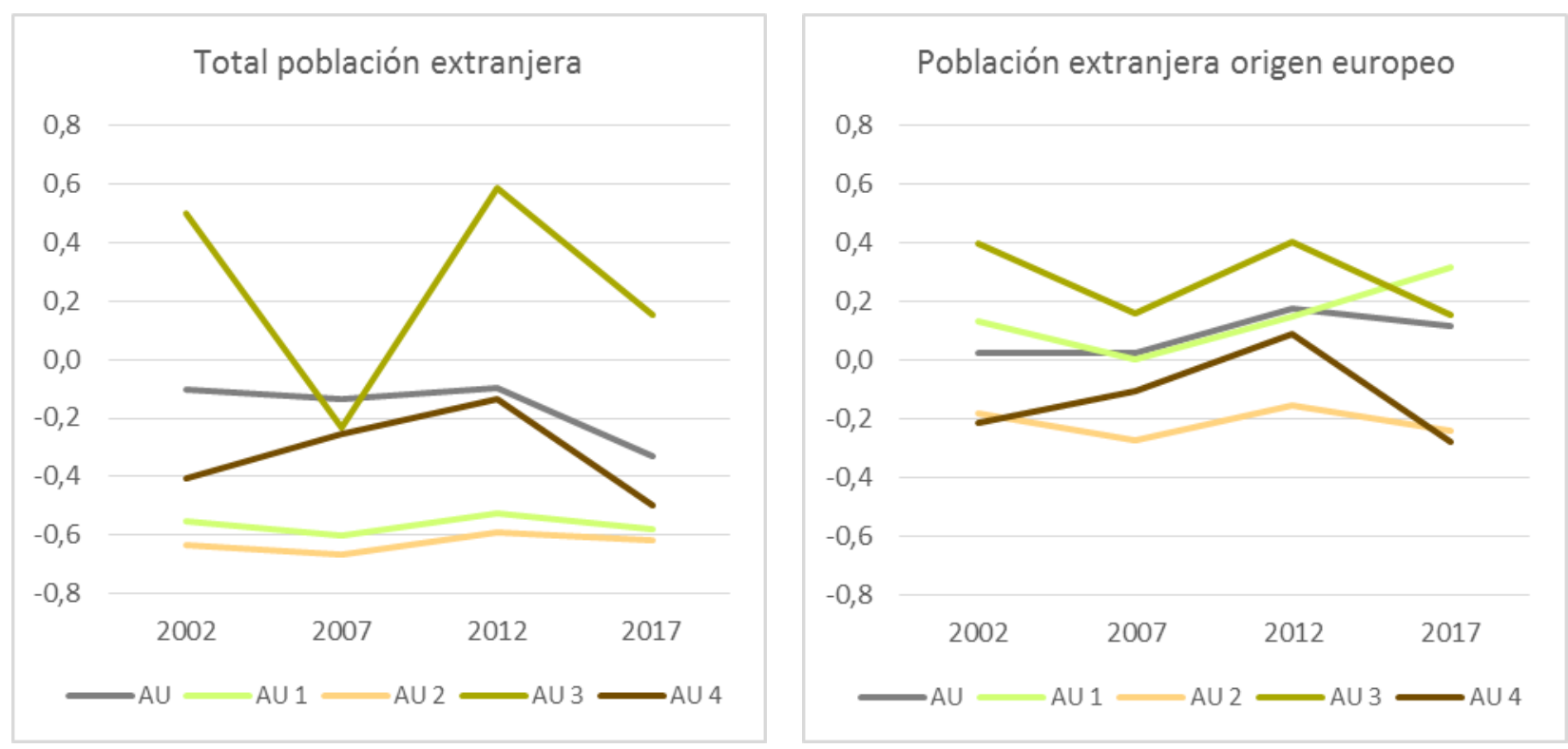

Fuente: elaboración propia a partir de los datos del Padrón Municipal de Habitantes y del Atlas Estadístico de las Áreas Urbanas en España

Los resultados muestran que la relación entre la tasa de envejecimiento y la de población extranjera es ligeramente negativa en el conjunto de las áreas urbanas en los cuatro años analizados. Esto es, a mayor proporción de extranjeros en las áreas, menor envejecimiento, tal como era esperable encontrar. Sin embargo, esta relación es muy débil (salvo en 2017, cuando aumenta su intensidad) en todos los años analizados, habida cuenta que la inmigración internacional no se ha distribuido de manera homogénea por las áreas urbanas del país. Los matices surgen al comparar los coeficientes de cada uno de los cuatro grupos de áreas urbanas que representan un modelo de envejecimiento. Las áreas urbanas de los modelos 1 y 2, aquellos que partían de una tasa de envejecimiento baja en 2002, presentan una relación negativa clara de intensidad moderada y estable en el período analizado. Se trata de áreas urbanas ubicadas 
en los archipiélagos, el litoral andaluz, más algunas del interior peninsular con vitalidad económica, áreas de claro destino de migrantes económicos y, en algunos casos, de migrantes jubilados de origen europeo.

Las áreas urbanas del modelo 4, el más negativo desde el punto de vista del envejecimiento demográfico, muestran una relación negativa pero poco intensa con la tasa de población extranjera, especialmente en el año 2012. Recordemos que en este grupo están incluidas las áreas gallegas, las del Cantábrico, y gran parte de las áreas de Castilla-León, entre otras. Las áreas urbanas del modelo 3, que partían de niveles de envejecimiento elevados, se caracterizan por una relación oscilante, de picos de sierra, y que contrasta con los anteriores modelos porque, salvo en 2007, año más cercano a la crisis económica, es positiva y de intensidad moderada. Esto es, en las urbes del modelo 3 envejecimiento y población extranjera van de la mano, y los picos de un año a otro están reflejando el efecto de la coyuntura económica.

El coeficiente de correlación entre tasa de envejecimiento y la población extranjera de origen europeo, una migración en la que abundan las personas jubiladas con una edad media relativamente elevada, muestra como es lógico el cambio de sentido a positivo: en el conjunto de las áreas urbanas, a mayor tasa de envejecimiento mayor peso de la población extranjera de origen europeo, aunque la intensidad de la relación es muy débil. Es en las áreas urbanas del modelo 3, entre las que tenemos ciudades del interior peninsular y, sobre todo, ciudades de la costa mediterránea conocidas por su importancia como lugar de residencia de estos inmigrantes -Torrevieja, Denia-ávea, etc.-, donde más intensa es la relación, aunque oscilante de unos años a otros. Es asimismo interesante constatar que las áreas urbanas del modelo 1, entre las que se encuentran las de las Islas Baleares, pero también hasta nueve más de la costa mediterránea, algunas con un perfil de migrante económico, se suman a esta relación positiva, de poca intensidad, aunque de tendencia ascendente. Por el contrario, las áreas urbanas del modelo 2 y del modelo 4 siguen mostrando una relación de sentido negativo, aunque en los dos casos de poca intensidad.

Los coeficientes de correlación entre el sobrenvejecimiento y población extranjera y población extranjera de origen europeo de las áreas urbanas calculados en este caso de acuerdo con los modelos específicos de sobrenvejecimiento encontrados, muestran que la relación entre la tasa de personas de 80 y más años y la presencia de población extranjera es negativa en los cuatro años estudiados, esto es, ahí donde encontremos sobrenvejecimiento hay menor presencia de 
población extranjera, o si se prefiere, donde más presencia de población extranjera haya, menor será el sobrenvejecimiento (Figura 17).

Figura 17. Coeficientes de correlación tasas sobrenvejecimiento (80 y más años) y de población extranjera de las áreas urbanas españolas (2002-2017)
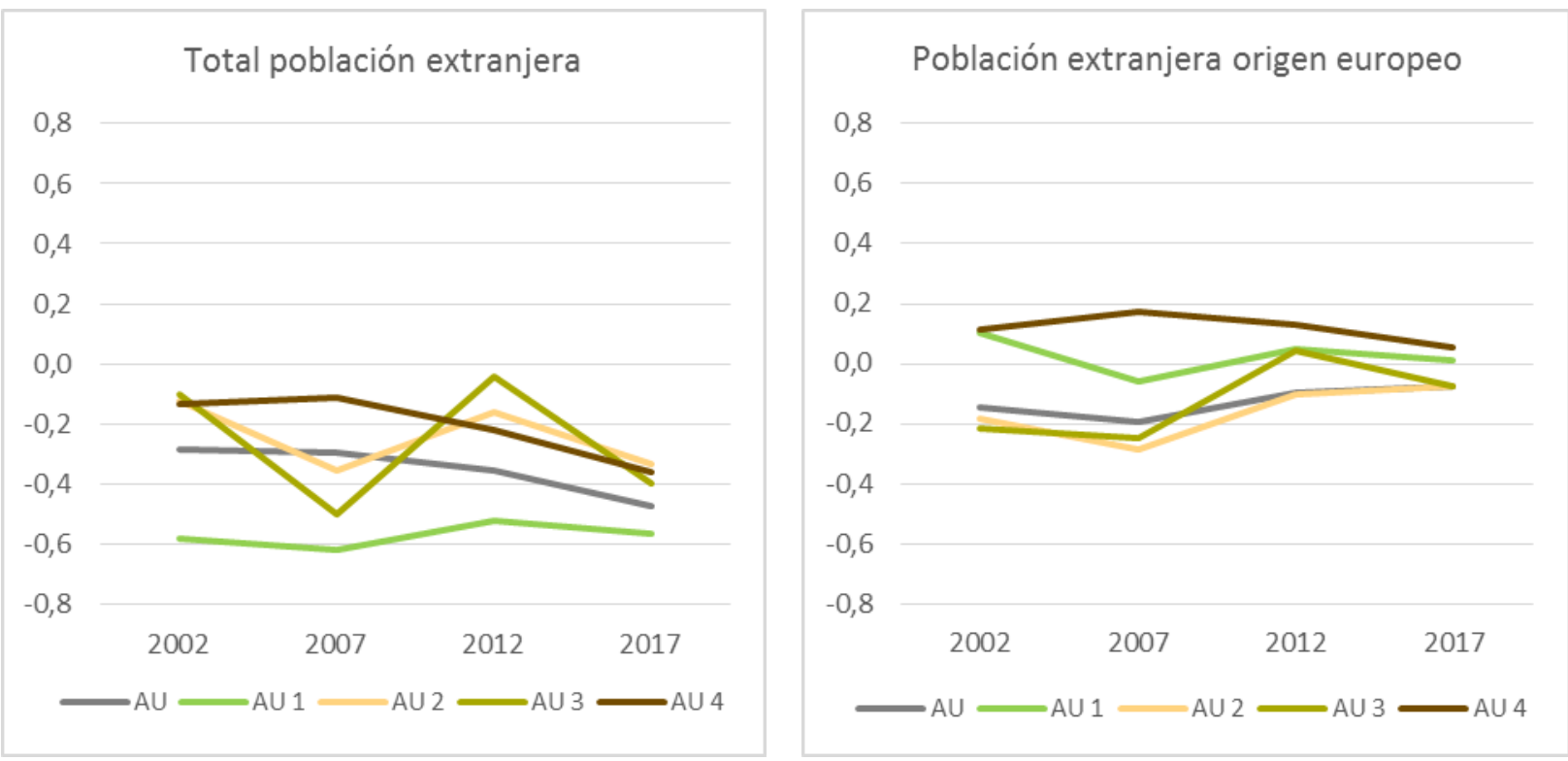

Fuente: elaboración propia a partir de los datos del Padrón Municipal de Habitantes y del Atlas Estadístico de las Áreas Urbanas en España

En concreto, las áreas urbanas incluidas en el modelo 1 -las de menor sobrenvejecimiento y mayor vitalidad demográfica- son las que reflejan una relación más intensa y, también más estable, y las áreas urbanas del modelo 3, la relación más oscilante, aunque de menor intensidad. Sin embargo, al centrar los cálculos de los coeficientes en la población extranjera de origen europeo, la relación se revela como menos homogénea: en el conjunto de las áreas urbanas pierde intensidad, aunque mantiene su carácter negativo, y lo mismo sucede en las áreas urbanas del modelo 2 y 3. Por el contrario, en las áreas urbanas del modelo 1 encontramos que en la mayor parte del período no hay relación entre sobrenvejecimiento y presencia de población extranjera; y en las áreas urbanas del modelo 4 la relación es positiva, aunque muy poco marcada.

\section{Conclusiones}

En este estudio se ha tratado de aportar luz sobre el envejecimiento de la población urbana en España, que siendo una cuestión de marcado interés demográfico y -sobre todo- social, dadas las implicaciones y consecuencias que se derivan del mismo, apenas cuenta con trabajos 
científicos dedicados específicamente a esta cuestión. Esto no significa que no sean temas tratados por los expertos en Geografía de la Población o en Demografía, sino que casi siempre lo son de manera conjunta, bien porque abordan el fenómeno del envejecimiento poblacional del país (incluyendo tanto la población rural como la urbana), bien porque se centran en esta última, pero sin prestar atención directa a los mayores, sino a diferentes variables demográficas, entre la que está -por supuesto- la dimensión etaria.

Cuando se encuentran estudios que abordan de forma específica el envejecimiento en las ciudades españolas, la escala de trabajo suele ser local, es decir, se trata de estudios de caso (Alegre Vicente, 2012; Del Valle Ramos, 2007), o que están centrados en los problemas y soluciones que suscita el envejecimiento urbano ("urbanismo gerontológico", servicios sociales, vivienda, transporte y movilidad, etc.), por poner solo algunos ejemplos (Bosch Meda, 2013; López Colás, 2007).

Por tanto, la principal novedad de este trabajo es contribuir al conocimiento actual del envejecimiento demográfico en el conjunto de las áreas urbanas españolas, explicando sus cambios recientes. Para ello se han definido una serie de modelos que ayudan a entender las diferencias evolutivas de las ciudades y a explorar -al menos de forma preliminar- una serie de variables implicadas, como la inmigración extranjera.

A grandes rasgos, se puede afirmar que el envejecimiento de la población urbana en España ha sido un fenómeno generalizado en los últimos años, del que apenas unas pocas ciudades han podido escapar. De hecho, la intensidad del mismo ha propiciado que la diferencia de edades con las áreas rurales (que de media están todavía más envejecidas) se haya visto reducida considerablemente. Por otro lado, la tendencia a la convergencia en las tasas de envejecimiento entre lo rural y lo urbano contrasta con la evolución de las tasas de sobrenvejecimiento, donde se está acentuando su carácter rural en este mismo período como corolario lógico de los procesos de despoblación, de las ganancias en años de vida y de envejecimiento in situ de las personas mayores.

Entrando más en detalle, se pueden matizar estas afirmaciones, ya que -como se ha explicadotanto desde el punto de vista temporal, como territorial la evolución no ha sido tan homogénea. En la primera etapa estudiada (2002-2007) se produce un ligero rejuvenecimiento de la población urbana, pero a partir de ahí el crecimiento en el porcentaje de personas mayores se torna nuevamente positivo. 
Por otra parte, al analizar territorialmente este fenómeno se aprecian diferencias muy relevantes. Si bien es cierto que hay un envejecimiento generalizado, no todas las áreas urbanas parten de la misma situación, ni evolucionan al mismo ritmo. Para discriminar las diferentes situaciones se propone un modelo explicativo que combina ambas variables, de tal forma que cada área urbana se incluye en una de los cuatro posibles tipos que se han definido, según se sitúe por encima o por debajo de la media de envejecimiento de las áreas urbanas españolas en 2002 y de la media de la variación de la tasa entre 2002 y 2017.

La primera tipología está formada por las áreas urbanas que partían de un envejecimiento menos intenso y que, además, se han mantenido en un ritmo de envejecimiento bajo a lo largo de los 15 años estudiados. La localización de estas ciudades es preferentemente mediterránea y su perfil es de áreas urbanas de tamaño pequeño y mediano. Las más relevantes son Murcia, Palma de Mallorca y Granada.

La siguiente clase se caracteriza por tener una situación relativamente buena de partida, aunque su evolución ha resultado preocupante al envejecer por encima de la media del país. A este grupo pertenecen, entre otras, el área urbana de Madrid, las ciudades de la mitad occidental de Andalucía y todas las del archipiélago canario.

En tercer lugar, encontramos áreas urbanas que, si bien partían de un considerable nivel de envejecimiento, se han mantenido con el paso de los años en unos niveles de variación del envejecimiento inferiores al conjunto de las ciudades españolas. Casi todas ellas se localizan en el cuadrante noreste peninsular y destaca sobre todas el área urbana de Barcelona.

Finalmente, las ciudades que se encuentran en peor situación son aquellas que no solo estaban más envejecidas, sino que además han acelerado más esa situación en los últimos tres quinquenios. El noroeste de la Península y -como excepción destacada- el área urbana de Valencia son las localizaciones preferentes de este grupo.

Este estudio presenta varias limitaciones. La primera, de tipo temporal: el período de 15 años analizado tiene la virtualidad de recoger un momento muy especial en la historia reciente migratoria española que tiene efectos en el proceso de envejecimiento demográfico; sin embargo, como proceso en desarrollo, convendrá estar atentos a los cambios en los próximos años que pueden incidir en el envejecimiento demográfico de las áreas urbanas españolas. Otra limitación está relacionada con algunas características de la principal fuente empleada, la Estadística del Padrón Continuo. Se es consciente que el Padrón no refleja adecuadamente la presencia de europeos jubilados residentes en España; también, que el hecho de recoger la 
población según su nacionalidad requiera asumir que la extranjera equivale a la población inmigrante; finalmente, hubiera sido más interesante al analizar la relación entre la tasa de envejecimiento y la población extranjera de origen europeo poder seleccionar los europeos de determinados países de origen, en vez del conjunto de europeos.

En cualquier caso, a pesar de estas y otras cuestiones en las que convendrá profundizar en futuros estudios, el análisis realizado en este trabajo pone de manifiesto el interés del fenómeno del envejecimiento urbano y las importantes consecuencias sociales y económicas que tendrá en las próximas décadas, debido a la magnitud que alcanza el mismo en las ciudades españolas.

Agradecimientos: Esta investigación ha sido posible gracias al proyecto coordinado lusoespañol «Ser mayor y vivir en un barrio con barreras topográficas en España y Portugal. Las infraestructuras de movilidad urbana vertical como elementos integradores e inclusivos para los ancianos (MOVE-AGED)» financiado por la Fundación General CSIC (FGCSIC), el Centro Internacional sobre el Envejecimiento (0348_CIE_6_E POCTEP) y el Fondo Europeo de Desarrollo Regional (FEDER).

Declaración responsable: Las/os autoras/es declaran que no existe ningún conflicto de interés con relación a la publicación de este artículo. Las tareas se han distribuido de la siguiente manera: el artículo ha sido coordinado por C. Montoro. Las/os dos autoras/es han participado en la revisión bibliográfica, la redacción del artículo y la elaboración de las figuras, estando la cartografía a cargo de J.J. Pons y los gráficos de dispersión a cargo de C. Montoro. 


\section{Bibliografía}

Abellán García, A., Ayala García, A., \& Pujol Rodríguez, R. (2017). Un perfil de las personas mayores en España, 2017. Indicadores estadísticos básicos (Informes Envejecimiento en red no. 15). Madrid. Retrieved from http://envejecimiento.csic.es/documentos/documentos/enredindicadoresbasicos17.pdf

Alegre Vicente, E. (2012). El envejecimiento de la población y sus repercusiones en el espacio urbano de la ciudad de Zaragoza. Espacio, Tiempo y Forma. Serie VI, Nueva época. Geografía, 4-5, 27-44. Retrieved from

http://revistas.uned.es/index.php/ETFVI/article/view/13721/12410

Antczak, E., \& Lewandowska-Gwarda, K. (2019). How Fast Is Europe Getting Old? Analysis of Dynamics Applying the Spatial Shift-Share Approach. Sustainability, 17(20), 5661. https://doi.org/10.3390/su11205661

ARUP - Help Age International - Intel - Systematica (2015). Shaping ageing cities: 10 European case studies. London. Retrieved from

https://www.arup.com/publications/research/section/shaping-ageing-cities

Bayona, J., Gil-Alonso, F., Rubiales, M. \& Pujadas, I. (2018). New spatial mobility patterns in large Spanish cities: from the Economic Boom to the Great Recession. Applied Spatial Analysis and Policy, 17(2), 287-312. https://doi.org/10.1007/s12061-017-9222-x

Boix Domènech, R. (2007). Concepto y delimitación de áreas metropolitanas: una aplicación a las áreas metropolitanas de España. In Seminario Las grandes áreas metropolitanas españolas en una perspectiva comparada. Sevilla, April $11 . \quad$ Retrieved from https://www.uv.es/ raboixdo/references/2007/07002.pdf

Casterline, J. B. (2003). Demographic transition. In P. Demeny \& G. McNicoll (Eds.), Encyclopedia of population, 1, 210-216. New York, NY: Thomson \& Gale.

Collantes, F., Pinilla, V., Sáez, L.A. \& Silvestre, J. (2010). El impacto demográfico de la inmigración en la España rural despoblada. Documentos de Trabajo Real Instituto Elcano, 30, 128. Retrieved from

http://www.realinstitutoelcano.org/wps/wcm/connect/77072c804408eba09153f77015846f3 f/DT30-

2010_Collantes_Pinilla_S\%C3\%A1ez_Silvestre_impacto_demografico_inmigracion_espana_rural_ despoblada.pdf?MOD=AJPERES\&CACHEID=77072c804408eba09153f77015846f3f 
Collantes, F., Pinilla, V., Sáez, L.A., \& Silvestre, J. (2014). Reducing depopulation in rural Spain: the impact of immigration. Population, Space and Place, 20(7), 606621. https://doi.org/10.1002/psp. 1797

Cutillas Orgilés, E. (2009). El espacio social de Torrevieja en el contexto geodemográfico valenciano. Boletín de la Asociación de Geógrafos Españoles, (49), 209-236. Retrieved from https://bage.age-geografia.es/ojs//index.php/bage/article/view/782

de Cos Guerra, O. \& Reques Velasco, P. (2006). Modernización económica y cambios demográfico-territoriales en España (período 1900-2001). Revista de Demografía Histórica, 24(1), 25-56. Retrieved from http://www.adeh.org/?q=es/node/6360

Delgado Urrecho, J. M., \& Martínez Fernández, L. C. (2016). La huella de la inmigración extranjera en las comunidades autónomas españolas de menor crecimiento demográfico. Cuadernos Geográficos, 55(2), 127-150. Retrieved from hittps://www.redalyc.org/pdf/171/17149048006.pdf

Delgado Urrecho, J.M., \& Martínez Fernández, L.C. (2017). Envejecimiento y desequilibrios poblacionales en las regiones españolas con desafíos demográficos. Ería, 1, 2143. https://doi.org/10.17811/er.1.2017.21-43

Del Valle Ramos, C. (2007). Envejecimiento demográfico y migraciones en la ciudad de Sevilla (2000-2004). Papeles de Geografía, 45-46, 187-201. Retrieved from https://revistas.um.es/geografia/article/view/43371

Díaz Hernández, J., \& Domínguez Mújica, J. (2014). De la base a la cúspide de la pirámide de población: el proceso de envejecimiento demográfico de Canarias en el horizonte de 2020. XXI Coloquio de Historia Canario-Americana (2014), XXI-066. Retrieved from hitp://coloquioscanariasmerica.casadecolon.com/index.php/aea/article/view/9548

Durán Muñoz, R. (2012). Atractivo de España para los jubilados europeos: del turismo a la gerontoinmigración. Panorama social, 16, 151-165. Retrieved from hittps://www.funcas.es/articulos/atractivo-de-espana-para-los-jubilados-europeos-delturismo-a-la-gerontoinmigracion/

ESPON/ACPA (2019). Adapting European Cities to Population Ageing: Policy challenges and best practices Final report. Retrieved from https://www.espon.eu/sites/default/files/attachments/1.\%20ACPA\%20Main\%20report.p $\underline{d f}$ 
EUROSTAT (2017). People in the EU - statistics on an ageing society. Retrieved from https://ec.europa.eu/eurostat/statistics-explained/index.php?title=People_in_the_EU_statistics_on_an_ageing_society

EUROSTAT (2020). Population on 1 January by broad age group and sex. Retrieved from https://ec.europa.eu/eurostat/data/database

Feria Toribio, J. M. (2004). Problemas de definición de las áreas metropolitanas en España. Boletín de la Asociación de Geógrafos Españoles, (38), 85-100. Retrieved from https://bage.age-geografia.es/ojs/index.php/bage/article/view/483

García Ballesteros, A., \& Jiménez Blasco, B. C. (2016). Envejecimiento y urbanización: implicaciones de dos procesos coincidentes. Investigaciones geográficas, (89), 58-73. Retrieved from http://www.scielo.org.mx/pdf/igeo/n89/0188-4611-igeo-89-00058.pdf

García Docampo, M \& Otero, R. (2012). Transición territorial: modelo teórico y contraste con el caso español, Revista Española de Investigaciones Sociológicas, REIS, 139, 133162. https://doi.org/10.5477/cis/reis. 139.133

García Jiménez, M., \& SCHRIEWER, K. (Eds.) (2009). Ni turistas ni migrantes. Movilidad residencial europea en España. Murcia: Ediciones Isabor.

Garrocho, C., \& Campos, J. (2015). Segregación socioespacial de la población mayor en la Ciudad de México, 2000-2010. In La situación demográfica de México (pp. 167-195). Retrieved from http://www.conapo.gob.mx/es/CONAPO/La_Situacion_Demografica_de_Mexico_2015

Gil-Alonso, F., \& Bayona-i-Carrasco, J. (2012). La dinámica urbana en España: evolución y tipología. Papeles de Geografía, 55-56, 95-108. Retrieved from https://revistas.um.es/geografia/article/view/176231/148711

Goerlich Gisbert, F. J., Mas Ivars, M., Azagra Ros, J., \& Chorén Rodríguez, P. (2007a). Actividad y territorio: un siglo de cambios. Bilbao: Fundación BBVA.

Goerlich Gisbert, F.J., Mas Ivars, M., Azagra Ros, J., \& Chorén Rodríguez, P. (2007b). La localización de la población española sobre el territorio: un siglo de cambios. Un estudio basado en series homogéneas (1900-2001). Bilbao: Fundación BBVA.

Goerlich Gisbert, F.J., Ruiz González, F., Chorén Rodríguez, P., \& Albert Pérez, C. (2015). Cambios en la estructura y localización de la población. Una visión de largo plazo (1842-2011). Bilbao: Fundación BBVA. 
INE, Instituto Nacional de Estadística (2018). Estadística del Padrón Continuo a fecha 1 de enero de 2017. Retrieved from

http://www.ine.es/dynt3/inebase/es/index.htm?type=pcaxis\&file=pcaxis \&path=\%2F+20\%2Fe245\%2Fp07\%2F\%2Fa2017

Gurría Gascón, J.L., Reques Velasco, P., \& Rodríguez Rodríguez, V. (2008). Le vieillissement de la population espagnole et les défis sociaux futurs. Sud-ouest Européen: Revue Géographique des Pyrénées et du Sud-Ouest, 26, 57-70. Retrieved from https://www.persee.fr/doc/rgpso_12764930_2008_num_26_1_5079

INE, Instituto Nacional de Estadística (2019). Cifras de Población a 1 de enero de 2019. Estadística de Migraciones. Año 2018 Datos Provisionales. Retrieved from https://www.ine.es/prensa/cp_e2019_p.pdf

INE, Instituto Nacional de Estadística (2018). Cifras de Población a 1 de enero de 2018. Estadística de Migraciones. Año 2017 Datos Provisionales. Retrieved from https://www.ine.es/prensa/cp_e2018_p.pdf

INE-Instituto Nacional de Estadística (2017). Cifras de Población a 1 de enero de 2017. Estadística de Migraciones. Año 2016 Datos Provisionales. Retrieved from https://www.ine.es/prensa/cp_2017_p.pdf

Lauf, S., Haase, D., \& Kleinschmit, B. (2016). The effects of growth, shrinkage, population aging and preference shifts on urban development-A spatial scenario analysis of Berlin, Germany. Land Use Policy, 52, 240-254.

Lesthaeghe, R. (2014). The second Demographic Transition: A concise overview of its development. Proceedings of the National Academy of Sciences, 111, 1811218115. https://doi.org/10.1073/pnas. 1420441111

Lewandowska-Gwarda, K., \& Antczak, E. (2020). Urban Ageing in Europe-Spatiotemporal Analysis of Determinants. ISPRS International Journal of Geo-Information, 9(7), 413.

Ministerio de Fomento (2017). Atlas Digital de las Áreas Urbanas en España 2017. Retrieved from http://atlasau.fomento.gob.es/

López Colás, J. (2007). Repercusiones urbanas del envejecimiento de la población en España. In Papers de Demografia, 315. Bellaterra: Centre d'Estudis Demogràfics. Retrieved from https://ddd.uab.cat/record/220844 
López Villanueva, C., Pujadas Rúbies, I., \& Rubiales Pérez, M. (2019). Hogares unipersonales y curso de vida: diversificación por edades y concentración espacial en las regiones urbanas de Madrid y Barcelona, Estudios Geográficos, 80(287), e012. https://doi.org/10.3989/estgeogr.201929.009

Montoro, C., López, D., \& Pons, J.J. (2009). Extranjeros y rejuvenecimiento demográfico: cambios recientes en la estructura demográfica de los casos históricos de las ciudades españolas. In L. López Trigal, A. Abellán García \& D. Godenau (Coords.), Envejecimiento, despoblación y territorio (pp. 253-266). León: Área de Publicaciones de la Universidad de León.

Morén-Alegret, R., \& Solana, M. (2004). Foreign immigration in Spanish rural áreas and small towns: current situation and perspectives. Finisterra, 39, 21-38. Retrieved from https://revistas.rcaap.pt/finisterra/article/view/1559

Nader, B., Prandato, L., Mobillion, V., Bochaton, A., Charreire, H., \& Baron, M. (2018). Vieillissement de la population et ville durable: quels enjeux? Ageing population and sustainable city: what are the challenges? Pollution Atmosphérique,

238). https://doi.org/10.4267/pollution-atmospherique.6664

Otero-Enríquez, R., García-Abad, J., Domínguez-Mujica, J., \& Pérez-Caramés, A. (2019). Inmigración y dinámicas territoriales en España. Crisis y recuperación (2008-2017). Anuario CIDOB de la Inmigración 190-

217). https://doi.org/10.24241/AnuarioCIDOBInmi.2019.190

Pérez Díaz, J. (2010). El envejecimiento de la población española. Investigación y ciencia, 410, 34-42. Retrieved from

http://digital.csic.es/bitstream/10261/29071/1/2010Inv\%2BCiencia.pdf

Pérez Díaz, J., \& Abellán García, A. (2018). Envejecimiento demográfico y vejez en España. Panorama social, 28, 11-47. Retrieved from https://www.funcas.es/publicaciones_new/Sumario.aspx?ldRef=4-15028

Pons, J.J., \& Montoro, C. (2020). El envejecimiento de los barrios urbanos en España: una propuesta de medición. In J.D. Sempere Souvannavong, C. Cortés Samper, E. Cutillas Orgilés \& J.R. Valero Escandell (Eds.), Población y territorio. España tras la crisis (pp. 175-190). Granada: Comares.

Pujadas Rúbies, I., \& Bayona-i-Carrasco, J. (2017). Dinámicas demográficas recientes en las áreas urbanas españolas en un contexto de crisis. In J.D. Sempere Souvannavong \& E. Cutillas Orgilés 
(Eds.), La población en España. 40 años de cambio (1975-2015) (pp. 133-155). Alicante: Universidad de Alicante.

Pujadas Rúbies, I., Bayona-i-Carrasco, J., \& Rubiales Pérez, M. (2016). Movilidad residencial a edades avanzadas en España: una perspectiva metropolitana. Finisterra, LI, 102, 121142. https://doi.org/10.18055/finis4250

Ramiro Fariñas, D., Pujol Rodríguez, R., \& Abellán García, A. (2016, February 2). Una reflexión necesaria sobre el inicio de la vejez. In Blog Envejecimiento [en-red]. Retrieved from http://bit.ly/1LgjyFN

Reques Velasco, P. (2009). Transición demográfica y epidemiológica. Envejecimiento y territorio. In J. Sastre, R. Pamplona \& J.R. Ramón (Eds.), Biogerontología médica (pp. 275-297). Madrid: Ergon/Biblioteca de la Sociedad Española de Geriatría y Gerontología.

Reques Velasco, P. (2017). La transición territorial. Cambios en las estructuras demo-espaciales en España (1900-2011): un análisis de base municipal. In J.D. Sempere Souvannavong \& E. Cutillas Orgilés (Eds.), La población en España. 40 años de cambio (1975-2015) (pp. 67-132). Alicante: Universidad de Alicante.

Roca Cladera, J., Moix Bergadà, M., \& Arellano Ramos, B. (2012). El sistema urbano en España. Scripta nova: revista electrónica de geografía y ciencias sociales, 16(396), 134. https://doi.org/10.1344/sn2012.16.3424

Rodríguez, V., Casado, M․Á. \& Huber, A. (Eds.) (2005). La migración de europeos retirados en España. Madrid: CSIC.

Rodríguez, V., Lardiés, R., \& Rodríguez, P. (2010). La migración y el registro de los jubilados europeos en España (Análisis del Real Instituto Elcano ARI N²0). Madrid: Real Instituto Elcano. Retrieved from

http://www. realinstitutoelcano.org/wps/wcm/connect/168e3980413536089631f66d616c216 $\underline{0 / A R I 20}$

2010_Rodriguez_Rodriguez_Lardies_migracion_registro_jubilados_europeos_espana.pdf?MOD $=$ AJPERES\&CACHEID $=168 \mathrm{e} 3980413536089631 \mathrm{f6} 6 \mathrm{~d} 616 \mathrm{c} 2160$

Rodríguez Rodríguez, V., \& Casado-Díaz, M. ${ }^{a}$ A. (2002). La migración internacional de retirados en España: limitaciones de las fuentes de información, Estudios Geográficos, 63(248-249), 533558. https://doi.org/10.3989/egeogr.2002.i248-249.237 
Rojo-Pérez, F., \& Fernández-Mayoralas, G. (2018). La calidad de vida en la población mayor. Panorama Social, 28, 49-73. Retrieved from https://www.funcas.es/wpcontent/uploads/Migracion/Articulos/FUNCAS_PS/028art03.pdf

Roquer, S., \& Blay, J. (2017). Evolución demográfica e inmigración extranjera en las zonas rurales españolas: del boom a la crisis. In J.D. Sempere Souvannavong \& E. Cutillas Orgilés (Eds.), La población en España. 40 años de cambio (1975-2015) (pp. 157-180). Alicante: Universidad de Alicante.

Vidal Domínguez, M.J., Labeaga Azcona, J.M., Casado Durandez, P., Madrigal Muñoz, A., López Doblas, J., Montero Navarro, A., \& Meil Landwerlin, G. (2017). Informe 2016. Las Personas Mayores en España. Datos Estadísticos Estatales y por Comunidades Autónomas. Madrid: Ministerio de Sanidad, Servicios Sociales e Igualdad. Instituto de Mayores y Servicios Sociales (IMSERSO) Retrieved from http://www.imserso.es/interpresent5/groups/imserso/documents/binario/112017001_i nforme-2016-persona.pdf

Vinuesa Angulo, J. (1996). Dinámica de la población urbana en España (1857-1991), Ciudad y territorio: Estudios territoriales, 107-108, 185-216. Retrieved from hitps://recyt.fecyt.es/index.php/CyTET/article/view/84125/62135

World Health Organization (2002). Active Ageing: A Policy Framework. Geneva: WHO. Retrieved from https://www.who.int/ageing/publications/active_ageing/en/ 